\title{
Realizing the physics of motile cilia synchronization with driven colloids
}

\author{
Nicolas Bruot ${ }^{1}$ and Pietro Cicuta ${ }^{2}$ \\ ${ }^{\mathbf{1}}$ Institut Lumière Matière, UMR5306 Université Claude Bernard Lyon 1 - \\ CNRS, Université de Lyon, Institut Universitaire de France, 69622 Villeurbanne \\ cedex, France \\ ${ }^{2}$ Cavendish Laboratory, University of Cambridge, Cambridge, UK, CB3 0HE
}

Xxxx. Xxx. Xxx. Xxx. YYYY. AA:1-31

This article's doi:

$10.1146 /(($ please add article doi $))$

Copyright (C) YYYY by Annual Reviews. All rights reserved

First page note to print below DOI/copyright line.

\section{Keywords}

hydrodynamic synchronization; driven colloidal particles; motile cilia; metachronal wave.

\begin{abstract}
Cilia and flagella in biological systems often show large scale cooperative behaviors such as the synchronization of their beats in "metachronal waves". These are beautiful examples of emergent dynamics in biology, and are essential for life, allowing diverse processes from the motility of eukaryotic microorganisms, to nutrient transport and clearance of pathogens from mammalian airways. How these collective states arise is not fully understood, but it is clear that individual cilia interact mechanically, and that a strong and long ranged component of the coupling is mediated by the viscous fluid. We review here the work by ourselves and others aimed at understanding the behavior of hydrodynamically coupled systems, and particularly a set of results that have been obtained both experimentally and theoretically by studying actively driven colloidal systems. In these controlled scenarios, it is possible to selectively test aspects of the living motile cilia, such as the geometrical arrangement, the effects of the driving profile and the distance to no-slip boundaries. We outline and give examples of how it is possible to link model systems to observations on living systems, which can be made on microorganisms, on cell cultures or on tissue sections. This area of research has clear clinical application in the long term, as severe pathologies are associated with compromised cilia function in humans.
\end{abstract}




\section{Contents}

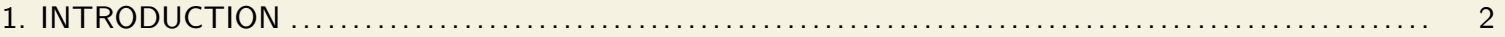

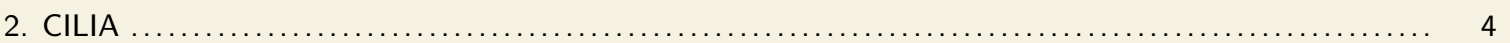

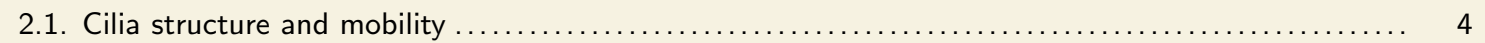

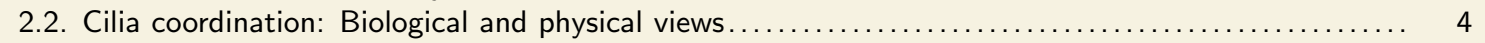

2.3. What can we learn from models: Relating the microscopics to collective dynamics .............. 7

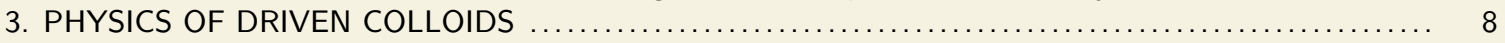

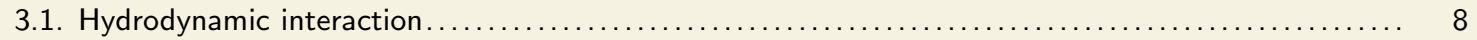

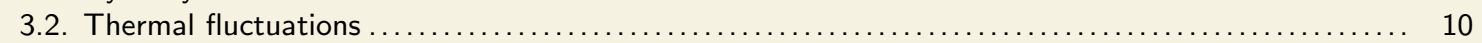

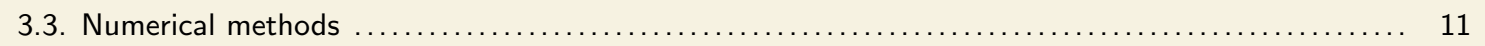

4. COLLECTIVE DYNAMICS AND METACHRONAL WAVE MODEL EXPERIMENTS ............... 11

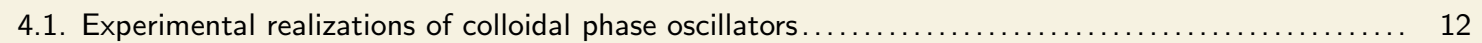

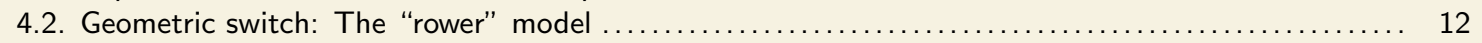

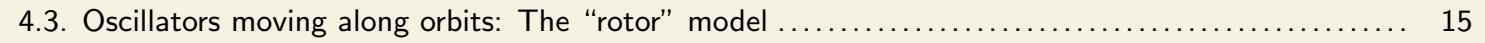

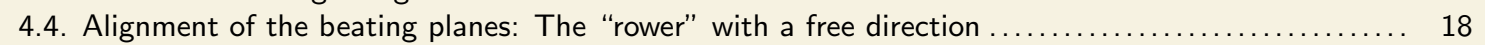

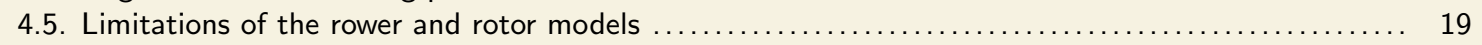

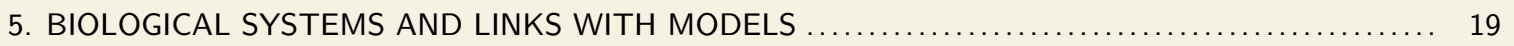

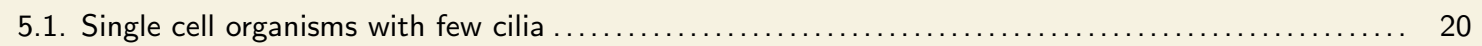

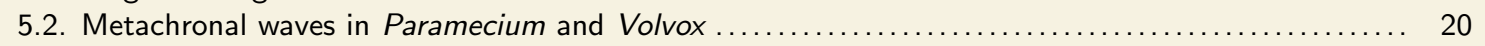

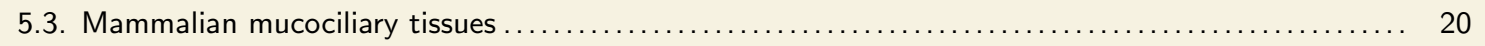

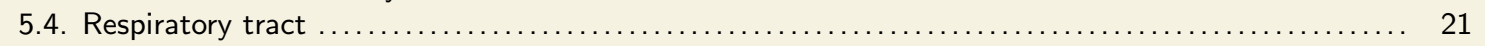

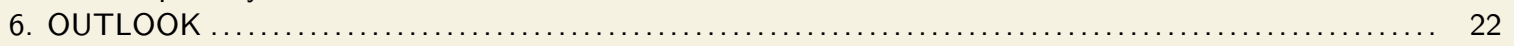

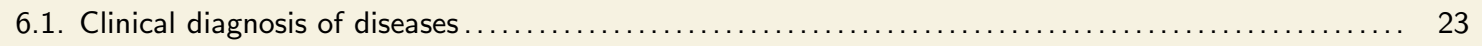

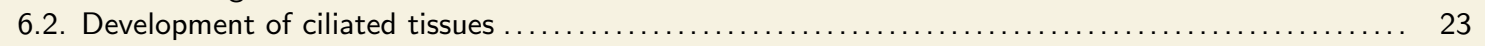

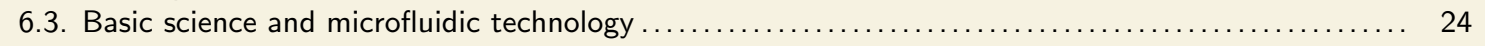

\section{INTRODUCTION}

Biological systems are often found to make use of "simple" and generic force-based mechanisms. Since most of life exists in a fluid state, an obvious medium for the transmission of force is the hydrodynamic interaction: the flow fields transmit forces between objects moving relative to each other or relative to the background fluid. At the microscopic cellular level, velocities and length scales combine to give a very low Reynolds number. Hydrodynamic coupling is an important feature in various biological flows, with key consequences in apparently diverse phenomena such as the motility of microorganisms (41, 50, 56, 60, 80, 81, 121), circulation in the brain (11) and functioning of the ear (79). In many cases motile cilia are involved in the generation of the flows, and the fluid acts as a medium that will couple the different beating cilia. A whole ciliated tissue can therefore be seen physically as a system of coupled oscillators. Because of the complexity of the beat pattern of a motile cilium and of its interaction with the surrounding fluid, simplified models of coupled oscillators have been developed by us and by other groups to address the question of the emergence of cooperative behaviors (41, 60). Such models have been very useful to describe the features of the coordinated patterns as a function of a limited number of control parameters.

We discuss here different models of oscillators that are used to gain insight in this topic, through experiment and theory, particularly to develop our understanding of the link between micro- and macroscopic dynamics. For example there is evidence that clusters of 

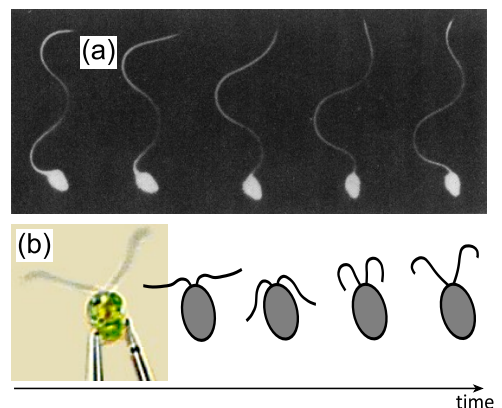

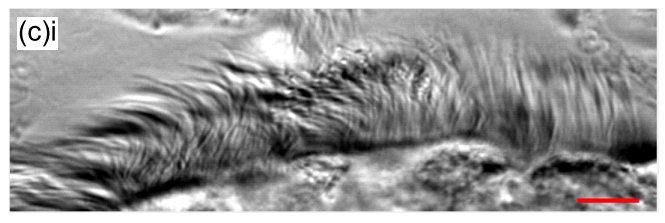

(c)ii

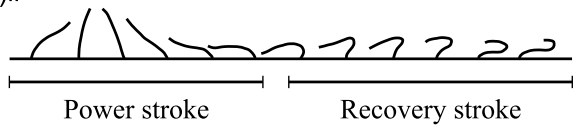

Figure 1

Flagella and cilia. (a) Flagellum of a sea urchin sperm cell showing a planar wave-like pattern (114). The snapshots are taken at $10 \mathrm{~ms}$ time intervals. (b) Biflagellated Chlamydomonas cell, held on a micropipette, with clearly visible flagella (adapted from (55)). Sketches illustrate the "breaststroke" phase-locked motion which takes place for the majority of time and corresponds to directed motion. (c)i Snapshot of side-on human airway epithelial cilia displaying a metachronal wave (scale bar: $5 \mu \mathrm{m}$ ). (c)ii Sketch respresentation of the time evolution of the shape of a cilium as in (c)i. The periodic cycle is made of a "power stroke", during which the cilium pushes the fluid, and a "recovery stroke", with the cilium coming back to its initial position at reduced drag. In a multicilated system, in presence of a metachronal wave, this sketch also represents the spatial distribution of cilia shapes as a snapshot in time.

close neighbours phase-lock into clear "dynamical motifs" (30), and classifying the main motifs under biologically relevant conditions will show which parameters are (or may be) optimized to sustain synchronization and flow over large scales in the biological context. Much remains to be explored in these well controlled models: fluids like mucus are complex and viscoelastic (54); flow occurs near soft tissues and with cilia close to each other, which is known to bring new hydrodynamic effects ranging from a shorter range coupling (135) to complex near-surface circulation patterns (130). Experiments on the colloidal models have provided unexpected results for example on the nontrivial consequence of thermal noise in coupled active systems (77) and on the role of feedback frequency (19).

The review is organized as follows. Section 2 introduces biological cilia, focusing on the origin of their activity that ultimately leads to the generation of a net flow. In section 3 we present the theoretical framework for the study of colloidal model oscillators, and in section 4 we describe the coarse-grained models of oscillators that have been implemented experimentally with particles driven by optical tweezers. To compare predictions of the simplified colloidal models to natural systems, it is relevant to also investigate real ciliated tissues and model microorganisms. This is discussed in section 5 with examples of increasing complexity: synchronization of the two flagella of Chlamydomonas reinhardtii, metachronal waves in Paramecium and Volvox, alignment of cilia in mammalian mucociliary tissues and observations on diseases affecting the respiratory tract. Finally, in section 6 we present our view on the implications of this work in the areas of clinical diagnosis of cilia-related diseases, in opening new possibilities in microfluidics for flow generation and control at the micrometric scale. The overarching (and largely outstanding) question addressed in this review is what determines the character of the dynamical steady state, and the related corollary of what has gone "wrong" in clinical conditions in which cilia coordination is observed to be compromised. 


\section{CILIA}

\subsection{Cilia structure and mobility}

Motile cilia are remarkably complex organelles, evolved early in life, whose structures is highly conserved throughout biology. Only some cells have motile cilia, either with the purpose of swimming, or to generate a flow. Mammalian sperm cells have a single, approximately $70 \mu \mathrm{m}$ long flagellum attached to the body (Figure 1(a)), while the Chlamydomonas alga has two flagella, about $12 \mu \mathrm{m}$ in length (2) (Figure 1(b)). In airway tissues (Figure 1(c)), cells are multiciliated and cilia form dense arrays $\left(2400 \mathrm{cilia} / \mathrm{cm}^{2}(93)\right)$; the filaments are much shorter, on average $7 \mu \mathrm{m}$ for human airways (84). While the length of cilia and flagella can vary significantly depending on the system, the internal structure of both cilia and flagella in eukaryotic cells is very conserved (131), leading to a fairly constant diameter of about $200 \mathrm{~nm}$. In most of the cases, when looking at a cross-section of a cilium, the internal structure (axoneme) consists in 9 microtubules doublets arranged on a circle in primary cilia, and 9 microtubules doublets in circle that surrounds two central single tubules in motile cilia (131) (Figure 2). The two structures are often called " $9+0$ " and "9 +2 " respectively. The microtubule doublets are responsible for the movement of motile cilia, and the orientation of the central tubules sets the plane of beating. In a cilium or flagellum, each doublet is a pair of dynein arms that slide against each other (10). This causes the whole cilium to bend, as shown in Figure 2(b). The energy required for the sliding is provided by adenosine triphosphate (ATP). The details of the motion of the filament are still being debated (87, 113), but it is well known that the outcome of all the activity, from the molecular up to the single cilium level, is an oscillating filament. Typical cycles are shown in Figures 1(a), (b) and (c)ii. For the short cilia in (b,c), the cycle starts with the "power stroke" with a fairly straight filament that changes its orientation, thus pushing the fluid. It returns to its initial position curled up, in the "recovery stroke" thus minimizing the interaction with the fluid. In the two strokes, a cilium has a very different drag coefficient because of the different conformations; this asymmetry makes the cycle non reciprocal, a necessary condition to provide a net force on the fluid over a period in a low Reynolds number environment. Cilia typically beat at a frequency of 5 to $100 \mathrm{~Hz}$ (118). Other beating cycles exist. For example, in uniflagellar spermatozoa, the cycle can be a wave-like planar (as in Figure 11(a)) or a helical pattern that propagates along the filament (10, 50, 137), pushing the cell forward. The planar pattern in Figure 1 (a) is somehow similar to the Sshape slithering motion of snakes, although the problem is more complex since the fluid is not immobile (121). Attempts have been proposed to account for the mechanisms at work within a cilium (69, 70), and to reproduce the cycle of oscillation (12, 13). Already at the level of a single cilium there are complex non-linear interactions amongst the constitutive components.

Physical considerations on cilia can be made on a single cilium. How the cilia units are structured and function is itself a fascinating question, which is well understood at the molecular scale, and is being addressed at the "system" level in terms of filament dynamics (49, 70, 81). However, a full model that includes in general the internal motor forces and cilia transfer of momentum to the surrounding fluid does not yet exist.

\subsection{Cilia coordination: Biological and physical views}

The function of motile cilia is essential in life: In complex organisms such as mammals, cilia are expressed in tissues to drive circulation in the brain, airways and fallopian tube. In these 
(a)

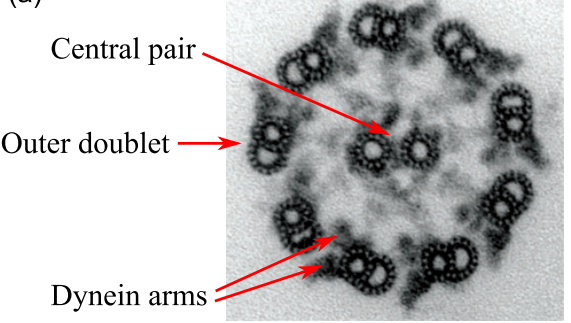

(b)

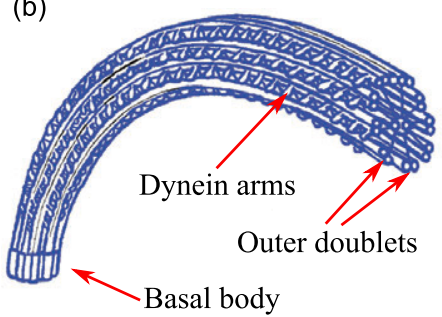

Figure 2

The "9+2" internal structure of a motile cilium is widely conserved in eukaryotes. (a) Axoneme cross-section showing nine microtubule doublets surrounding a central pair (87). (b) Flagellar axoneme extending from the basal body on which the cilium is anchored to the rest of the cell. The dynein arms are the motor of the cilium beat and induce the sliding of microtubules against each other (from neighbouring doublets), causing the overall bending of the filament (87).

organs the active cilia units interact with each other creating a coupled dynamical system, which can lead to coordinated beating in a "metachronal wave" (40, 63), see Figure 1. (c)i. This wave is particularly efficient at sustaining directed surface flow (10). Nearby cilia will phase-lock, and may for example beat in-phase or out of phase, or indeed may be in a condition where they can readily switch between the two dynamical states as in the algae Chlamydomonas reinhardtii (106).

Coordinated motion is crucial for the effective functioning of cilia and flagella, the elements of eukaryotic cells implicated in generating fluid flows and motility (10, 14, 40), and may be exploited in artificial conditions (3, 67, 115, 130) and low Reynolds number (Re) swimmers (83, 102, 108). Motile flagella and cilia interact through the velocity field in a low Re regime (63, 81, 99). The magnitude of the coupling forces is comparable to the random thermal forces, raising the question of how biological systems might exploit this competition between locking and random phase drift, or how architectures leading to stable synchronized states of multiple cilia can have evolved. Synchronization is a general phenomenon in nature (105), with many technological applications. Hydrodynamic synchronization at low Re is a well defined subset of this, in which coupling is viscous (181), noise (thermal and intrinsic contributions) is important (89, 106), and the details of the driving forces play a key role (99, 135). Recent progress in hydrodynamic synchronization is reviewed in (41, 60), and an overview of low Reynolds number (Re) flows is in (81).

One approach to understand hydrodynamic synchronization is to study systems of a few flagella. For example, the biflagellated alga Chlamydomonas (Figure 1(b)) has been a test-bed for experiments exploring synchronization of flagella (56), recently showing that hydrodynamic flows are sufficient to describe phase-locked cilia dynamics (17). Particlebased experimental and conceptual models, where the emerging dynamics in coupled arrays of driven colloidal phase oscillators can be probed, have shown clearly the role that geometry (18, 26, 30, 85), type of drive (20, 34, 126), and details of the driving potential (20, 76, 78, 127, 135) can have on the collective state. By enabling selective control of individual parameters, and analytical results in some limit conditions (26, 126, 127), these models form a solid foundation to build our understanding of natural systems such as mucociliary tissues. These models maintain the correct far-field form of the hydrodynamic flow caused by a cilium, can describe the presence of a nearby solid surface, and most im- 
(a)
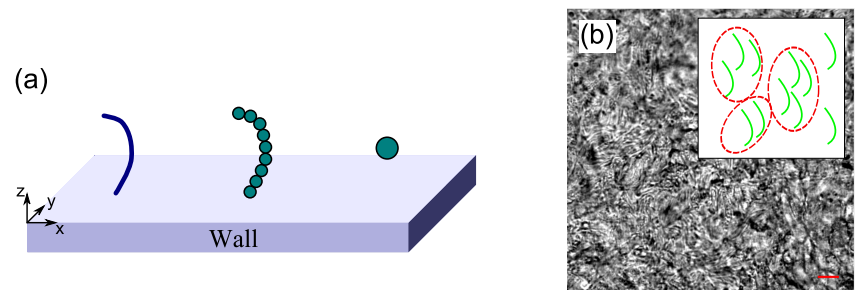

Figure 3

Hydrodynamic coupling leads to coordinated motion of cilia and can be studied using simpler models. (a) In a coarse-grained model, a single cilium can be modeled as a chain of spherical colloidal particles, and even as a single bead. Despite their simplicity, these descriptions approximate well (in the far field limit) the characteristics of the fluid flow generated by a full filament. (b) Top view of a culture of ciliated cells (scale bar: $10 \mu \mathrm{m}$ ). Movies of such cultures can be studied to look for local dynamical patterns (illustrated in the inset) that are predicted by model studies involving the hydrodynamic coupling.

Most eukaryotic cells have cilia, which may be motile or immotile. It is now thought that most cilia can act as chemical- or mechano-sensors, while only motile cilia enable epithelial cells in various tissues to generate surface flows (44). Motile cilia are highly conserved structures in the evolution of organisms, essentially the same in plants and animals; they generate the transport of fluid by periodic beating, through remarkably organized behavior in space and time. It is not clearly known how these spatio-temporal patterns emerge and what sets their properties. Individual cilia are nonequilibrium systems with many degrees of freedom, but can be represented by simpler effective force laws that drive oscillations, and paralleled with nonlinear phase oscillators often studied in physics. Recent models capture the key physical elements at work within each filament (69): each cilium undergoes periodically a forward "power stroke", followed by a backward "recovery stroke" in which it is more bent and closer to the surface, thus resulting in a net momentum transfer to the fluid over a cycle (Figure 1(c)ii). The source of synchronization observed in cilia and flagella is often thought to be of hydrodynamic origin, based on many observations (17, 22, 55, 63), but the details of coupling in the case of mucociliary tissues (and specifically of the airways) are not known and are a topic of current research. Synchronization in systems of many oscillators holds many open questions, and is close to frontiers of soft matter and statistical physics dealing more widely with the collective behavior of active elements, and the emergence of non-equilibrium structures (48, 90).

portantly can account for the physiological properties of the beating cilia (i.e. shape of the stroke, with power and recovery phases) by matching the properties of living system cilia cycles as the driving rule.

A fruitful approach to date has been to build experimental models of driven phase oscillators, sufficiently simple for both theoretical and experimental studies. Experimentally, these involve optically trapped colloidal sized spheres, which play the role of beating cilia, maintaining the same regime of length and time scales (Figure 3(a)). In an attempt to model (both experimentally and theoretically) the physics of hydrodynamic synchronization of cilia, two main ideas have emerged.

One model consists of a "geometric switch": the model cilium has two states corresponding to its back and forth motions. It displays a discontinuity in the driving force 
and is motivated by the existence of the power and recovery strokes (Figure 1)(c)ii) and by the fact that molecular motors undergo discrete attachment/detachment events which couple to the force generation. It was first studied theoretically in $(28,29,62)$, then by our group (26, 30, 77), and others (135).

In contrast with the configuration-controlled system above, a stress-controlled "rotor" model has also emerged (99). This has recently been studied very generally for two rotors by Uchida and Golestanian (126, 127), extending the case of circular orbits by Lenz (99). A series of important results has been obtained modelling cilia as rotors (99, 126, 128); in recent work (76, 127) it was shown that both the flexibility of the orbit, and the mean force profile, determine the strength of synchronization. By small adjustments of its physical parameters, it is possible to achieve control over the state of synchronization of the system.

Some of our results on the two models are discussed in Section 4 It is not yet established which of the two ideas (stress- or configuration-controlled oscillators) is most appropriate to describe a biological scenario.

The research frontier is now to develop the model experiments further, to include key biological aspects (such as the high density of cilia in some organs, or the presence of a nonNewtonian fluid), and to enable rules and properties at the micro-scale to be linked with the macroscopic dynamics. In this sense, understanding cilia synchronization in biological systems is an open and very important area of research. The recent progress gained in simple systems, coupled with advances in optical microscopy, ciliated cell culture, microfluidics, optical manipulation and camera technology, make this area ripe for experimental investigation.

\subsection{What can we learn from models: Relating the microscopics to collective dynamics}

Each biological cilium is itself a complex structure (see Figure 2), and its own regular beating (and switching) is constrained by a mechanical feedback (69, 113). In a given flow condition, the mechanical stress and the geometrical configuration are coupled parameters, and the feedback condition of the geometric switch is a simple way to account for how a cilium senses the moment to switch between so-called power and recovery strokes (63). It is clear that a complex scenario such as this requires models capable of joining up quite different length scales, from molecular to cilium level, and up to the interactions of cilia with the fluid and each other. In this spirit, and depending on the focus of the question, some aspects have to be suitably simplified and reduced.

Certain organisms (biological model systems) also allow, to some degree, to address specific aspects of such an entangled problem. For the alga Chlamydomonas, the synchronization of the beating filaments was shown recently $(47,55,57)$ and the visualization of cilia shape was also achieved (2). While Chlamydomonas has been very useful for developing and tuning physical models to biological systems, ultimately important goals lie in understanding human physiology, where ciliated tissues have large numbers of cilia, and cilia arrangement is likely to be important. In tissues such as the trachea of mammals, the average frequency of beating and speed of the wave can be measured readily, but observation of the individual beating cilia is hampered by the presence of optically opaque mucus (23, 136), see Figures 1(c)i and 6(b).

In this context, colloidal model experiments, where the parameters of the system can be tuned readily and visualization is straightforward, have an important role to play in 
elucidating the factors controlling emergent collective states. Colloidal particle driven systems (in a way, a model even simpler and more flexible than Chlamydomonas) can be used to demonstrate specific effects: for example thermal noise (capable of course of destroying the synchronization if sufficiently large) introduces a delay between the coupled oscillators (77). The conditions for phase locking can be predicted in simple scenarios (20), and we have developed design principles to explain the emergence of characteristic dynamical patterns in small networks of oscillators (26, 30). The systems we investigated to date can be thought of as idealized cilia synchronization experiments: The optical trap force plays the role of the molecular motors that induce active movement, and the hydrodynamic flow field produced by the moving beads well represents beating cilia, at least at large distances (81). These models are also general experimental systems to probe the physics of stochastic and actively forced hydrodynamically coupled oscillators. From the theoretical perspective, working with models enables to develop well defined general results, for example various models have been proposed recently addressing different aspects of metachronal waves (47, 65, 69, 81, 99, 135).

The role of models, where individual parameters can be tuned selectively, is to establish the general rules and links between the emergence and the properties of synchronized states (e.g. the collective phenotypes, like wave dynamics and the spatial or temporal coherence of coordination) and the key physical properties of the individual active structures (e.g. mechanics, activity and geometry of each motile cilium, and fluid rheology).

\section{PHYSICS OF DRIVEN COLLOIDS}

To gain an understanding in the synchronization of cilia and the emergence of metachronal waves, it is necessary to coarse-grain the filaments in various ways, and analyze the emergent behavior experimentally, numerically or with analytic calculations. In a quite accurate description of the drag with the fluid, the filament can be replaced by a chain of spherical beads or rods (27, 40, 73, 130) (Figure 3,a)). This can also be realized experimentally with magnetic particles self-assembling in chains (36, 129, 130). These models are useful to study the flow generated close to the filament and to obtain its shape during a beating cycle, but so far it has only been possible to actuate the filaments with a uniform external force. Elliptical particles have also been used (71). To consider questions relating to synchronization between different cilia, an even more coarse-grained model can be used: Since the filament can be seen as a point in the far field, it can be assumed that the fluid flow generated by the cilium is the same as the one created by a moving sphere. Hence, a cilium can be modelled as just a sphere, see Figure 3(a). Many recent models use this level of coarse-graining (99, 126, 128), as this greatly simplifies the calculation of drag forces, both those acting on the individual object, and the force induced by one object on another. The cycle shape and bead velocities contain in a coarse-grained fashion the cilium properties, and are at the core of the model. It is possible, as outlined in section 4 , to deploy these models (and also carry out experiments) without locking the phase of each cilium to an external driving clock.

\subsection{Hydrodynamic interaction}

This section recalls the main equations that govern colloidal models, and the numerical methods that can be used to simulate systems of hydrodynamically coupled oscillators.

One key interest of colloidal oscillators as a model of cilia is to provide simple ways to 
describe the hydrodynamic interaction. Studies until now we have assumed a Newtonian fluid in a low Reynolds' number configuration, that the propagation of the transfer of momentum from the fluid is instantaneous, and that the colloidal particles do not rotate. For a set of $N$ colloidal particles, the hydrodynamic coupling between the external forces acting on the colloids (all forces but the one from the fluid) and the resulting velocities of the particles is given by a relation of the form

$$
\mathbf{v}_{i}=\sum_{j=1}^{n} \boldsymbol{\mu}_{i, j} \mathbf{F}_{j}
$$

where $\mathbf{v}_{i}$ is the velocity of bead $i, \mathbf{F}_{j}$ the external force acting on bead $j$, and $\boldsymbol{\mu}$ is an $N \times N$ mobility matrix whose elements are $3 \times 3$ second-rank tensors. The choice of $\boldsymbol{\mu}$ depends on the presence or not of no-slip boundary conditions (a nearby wall) and on the level of approximations regarding the finite radius $a$ of the particles (near field effects). We outline below the most commonly used mobility matrices.

3.1.1. Far field, no wall: Oseen tensor. A moving particle in an unbounded quiescent fluid generates a flow $\mathbf{u}(\mathbf{r})$ as a result of the external forces acting on the particle. Such a solution of the Stokes equation (33, 68) for the fluid is called a Stokeslet (24). If a second particle is placed at $\mathbf{r}_{i}$ in the fluid it will simply follow the fluid at the velocity $\mathbf{u}\left(\mathbf{r}_{i}\right)$. In the case of $N$ particles, when the interparticle distances $r_{i, j}=\left|\mathbf{r}_{i}-\mathbf{r}_{j}\right|$ are large compared to their radius $a$, and when keeping only the leading orders in $a / r_{i, j}$, the mobility matrix simplifies to the Oseen tensor $\boldsymbol{\mu}=\mathbf{H}$. In that case, the diagonal terms simply represent the Stokes drag on a sphere, and the non-diagonal terms are directly related to the Stokeslets (33, 35):

$$
\mathbf{H}_{i, j}= \begin{cases}\frac{1}{8 \pi \eta r_{i, j}}\left(\mathbf{I}+\hat{\mathbf{e}}_{i, j} \hat{\mathbf{e}}_{i, j}\right) & \text { if } i \neq j \\ \frac{\mathbf{I}}{6 \pi \eta a} & \text { if } i=j\end{cases}
$$

with $\mathbf{I}$ the unit tensor and $\hat{\mathbf{e}}_{i, j}$ a unit vector in the direction defined by the two particles $i$ and $j$.

3.1.2. Near field, no wall: Rotne-Prager tensor. A more accurate description of the hydrodynamic interaction than the Oseen tensor accounts for the finite size of the particles in the non-diagonal terms of the mobility matrix. The correction, which appears as a higher order term in $a / r_{i, j}$ takes into account that a flow created by a particle is "reflected" on the other particles, thus affecting the velocity of the first particle (33, 68). To stay consistent with the expansion to the next order in $a / r_{i, j}$, it is also necessary to expand the Stokeslet flow of a single particle to the third order, as well as taking into account that a particle moving in an non-uniform flow undergoes a translational motion slightly different from the sole fluid velocity at the position of the particle (Faxén theorem for translation). All together, the corrections above lead to the Rotne-Prager mobility matrix (33)

$$
\boldsymbol{\mu}_{i, j}^{\mathrm{RP}}=\left\{\begin{array}{lc}
\frac{1}{6 \pi \eta a}\left[\frac{3 a}{4 r_{i, j}}\left(\mathbf{I}+\hat{\mathbf{e}}_{i, j} \hat{\mathbf{e}}_{i, j}\right)+\frac{1}{2}\left(\frac{a}{r_{i, j}}\right)^{3}\left(\mathbf{I}-3 \hat{\mathbf{e}}_{i, j} \hat{\mathbf{e}}_{i, j}\right)\right] & \text { if } i \neq j \\
\frac{\mathbf{I}}{6 \pi \eta a} & \text { if } i=j
\end{array} .\right.
$$


3.1.3. Presence of wall: Blake tensor. Many biological systems involve the hydrodynamic coupling between objects that are moving close to a wall, with a no-slip boundary condition. This is particularly true for ciliated tissues, where the filaments are anchored at the surface of the cells. The Blake tensor describes the interaction between spheres in a semi-infinite fluid with a no-slip boundary condition at the surface.

For the diagonal terms of the mobility matrix, the presence of the surface modifies the Stokes drag that can be expanded as a series in $a / z_{i}$, where we take a system of coordinates $(x, y, z)$ with $\hat{\mathbf{e}}_{z}$ normal to the surface and $z=0$ at the surface (104). An expansion to the sixth order has already been used to study the flow generated by arrays of artificial cilia (130). The corresponding diagonal terms read:

$$
\left\{\begin{array}{l}
\boldsymbol{\mu}_{i, i}^{x, x}=\boldsymbol{\mu}_{i, i}^{y, y}=\frac{1}{6 \pi \eta a}\left[1-\frac{9 a}{16 z_{i}}+\frac{1}{8}\left(\frac{a}{z_{i}}\right)^{3}-\frac{1}{16}\left(\frac{a}{z_{i}}\right)^{5}\right] \\
\boldsymbol{\mu}_{i, i}^{z, z}=\frac{1}{6 \pi \eta a}\left[1-\frac{9 a}{8 z_{i}}+\frac{1}{2}\left(\frac{a}{z_{i}}\right)^{3}-\frac{1}{8}\left(\frac{a}{z_{i}}\right)^{5}\right] \\
\boldsymbol{\mu}_{i, i}^{\alpha, \beta}=0 \text { for } \alpha \neq \beta .
\end{array}\right.
$$

For the non-diagonal terms, Blake proposed in (7) to describe the fluid flow created by a Stokeslet near a surface by an image method (as in electrostatics). In this method, the no-slip boundary condition at the wall is satisfied by describing the effect of the wall as equivalent to an infinite fluid, but with a second Stokeslet at the mirror position of the first Stokeslet and with an opposite force. For $N$ particles, this leads to the following expressions for the Blake mobility matrix (7, 107):

$$
\boldsymbol{\mu}_{i, j}^{\mathrm{B}}=\frac{1}{8 \pi \eta}\left[\mathbf{G}^{\mathrm{S}}\left(\mathbf{r}_{i}-\mathbf{r}_{j}\right)-\mathbf{G}^{\mathrm{S}}\left(\mathbf{r}_{i}-\overline{\mathbf{r}}_{j}\right)+2 z_{j}^{2} \mathbf{G}^{\mathrm{D}}\left(\mathbf{r}_{i}-\overline{\mathbf{r}}_{j}\right)-2 z_{j} \mathbf{G}^{\mathrm{SD}}\left(\mathbf{r}_{i}-\overline{\mathbf{r}}_{j}\right)\right] .
$$

with $\mathbf{r}_{i}=\left(x_{i}, y_{i}, z_{i}\right), \overline{\mathbf{r}}_{i}=\left(x_{i}, y_{i},-z_{i}\right)$ and with the elements of the Green functions given by (7, 107)

$$
\begin{aligned}
& \mathbf{G}_{\alpha, \beta}^{\mathrm{S}}(\mathbf{r})=\frac{\delta_{\alpha, \beta}}{r}+\frac{r_{\alpha} r_{\beta}}{r^{3}} \\
& \mathbf{G}_{\alpha, \beta}^{\mathrm{D}}(\mathbf{r})=\left(1-2 \delta_{\beta, z}\right) \frac{\partial}{\partial r_{\beta}}\left(\frac{r_{\alpha}}{r^{3}}\right) \\
& \mathbf{G}_{\alpha, \beta}^{\mathrm{SD}}(\mathbf{r})=\left(1-2 \delta_{\beta, z}\right) \frac{\partial}{\partial r_{\beta}} \mathbf{G}_{\alpha, z}^{\mathrm{S}}(\mathbf{r})
\end{aligned}
$$

with $\delta$ the Kronecker delta.

In the most general case, when the size of the particles $a$ is not negligible compared to $r_{i, j}$ or $z_{i}$, the Blake tensor can be corrected for the beads radii. In a derivation similar to the Rotne-Prager tensor, the Faxén theorem gives (51, 130)

$$
\boldsymbol{\mu}_{i, j}=\left(1+\frac{a^{2}}{6} \nabla_{\mathbf{r}_{i}}^{2}\right)\left(1+\frac{a^{2}}{6} \nabla_{\mathbf{r}_{j}}^{2}\right) \boldsymbol{\mu}_{i, j}^{\mathrm{B}},
$$

for $i \neq j$. The expanded version of this formula is heavy and is not printed here but can be found in the supplementary information of (130).

\subsection{Thermal fluctuations}

As a consequence of the small scales involved, small biological systems are subject to Brownian motion. The random force $\mathbf{f}(t)$ that acts on a spherical colloid is in practice well 
approximated by white noise and is characterized by (92):

$$
\left\{\begin{array}{l}
\langle\mathbf{f}(t)\rangle=0 \\
\left\langle\mathbf{f}(t) \cdot \mathbf{f}\left(t^{\prime}\right)\right\rangle=2 \nu \gamma k_{B} T \delta\left(t-t^{\prime}\right)
\end{array}\right.
$$

with $\gamma=6 \pi \eta a$, and $\nu \in\{1,2,3\}$ the dimension of the system.

In a system of several particles, this thermal fluctuating force has to be included in the forces $\mathbf{F}_{j}$ of the mobility matrix in Equation (1). It then leads to coupled terms of noise in the velocities $\mathbf{v}_{i}(25,92)$, which we have shown to induce non-trivial correlations in motile systems (77). Thermal noise also induces rotation of the particles and this rotation couples to the translation $(110)$.

\subsection{Numerical methods}

Numerical methods have provided a lot of insight into questions of fluid dynamics, beat efficiency, and generally in finding solutions for any model dynamical system that has been posed (40, 99, 124), since these can usually be treated analytically only in very special cases (19, 25, 126).

Brownian Dynamics (BD) codes include the treatment of thermal fluctuations. Such simulations are extremely useful to provide quantitative solutions to compare with experiments, and to extend theory approaches (which are often simplified, and typically neglect thermal noise). BD codes can be pushed to very large systems of over 100 fully coupled model cilia (this is fewer than the active elements in multiciliated tissues, but much larger than possible with any optical trap device), even with a standard desktop computer. Simulations of course assume that the hydrodynamic interactions are known mathematically: The equations outlined above do accurately describe coupled colloidal particles in Newtonian liquids, but the problem becomes more challenging when e.g. viscoelasticity is introduced.

In our own work we use a $\mathrm{C}++\mathrm{BD}$ program that implements the Ermak McCammon algorithm (42), providing a numerically fast way to integrate the equations of motion of $N$ particles interacting with the Oseen or Rotne-Prager tensors, with coupled thermal fluctuations. In this algorithm, momentum transfer is instantaneous (global) and noise has long-range spatial correlations.

\section{COLLECTIVE DYNAMICS AND METACHRONAL WAVE MODEL EXPERIMENTS}

Understanding the synchronization of cilia and flagella can be done by means of in vitro experimental observations, or by working on simplified physical models that describe the beating pattern of cilia. Since it is increasingly accepted that the hydrodynamic interaction plays a key role in the synchronization of these systems $(54,62,63,65)$, the models should include that coupling. This section focuses on colloidal models where the hydrodynamic interaction dominates. Other interactions, such as mechanical (53) through the cells, or steric repulsion might also be relevant.

The geometric switch and rotor models below are simple enough to carry out numerical simulations or analytical calculations by reducing the multiple degrees of freedom of the complex systems into a few control parameters which can be tuned. These help to understand theoretically the biological systems. They also make it easier to account for the hydrodynamic interaction. For example, sperm flagella have been first modelled by a 
waving sheet (39, 121) or by a $2 \mathrm{~d}$ or $3 \mathrm{~d}$ filament $(61,64,86)$, in order to determine the fluid flow they create. Experimental realizations of actuated filaments that can mimic the motion of a cilium also exist, at the micrometric (27, 72, 130), and macroscopic (117) scales.

\subsection{Experimental realizations of colloidal phase oscillators}

The colloidal particle experiments are based on the physical intuition that the complex structural details of the cilia might be coarse-grained into the details of how the colloidal particles are driven. Two classes of colloidal models (geometric switch and stress driven) have been developed in this vein, exploring in first instance the conditions for optimal coupling. We have also introduced a modified version of the geometric switch model with a free orientation so that collective alignment properties could be investigated. We present here these coarse-grained models and their most interesting collective features that have been observed by us and other groups.

Experimentally, optical tweezers can be used to impose external forces (in the range from fractions to hundreds of piconewtons) onto colloidal particles (dielectric spheres of size range between half and 5 micrometers, Figure 4(a)). In purpose-made optical tweezers setups like (76) it is possible to generate many laser beams (or achieve many traps by time sharing one beam across multiple positions, at much higher frequency than any dynamics in the system) by deflecting beams via surface light modulators or acousto-optical modulators. By analysing the positions of the colloidal particles at high frame rate (typically hundreds of times per second), and implementing a feedback loop, it is then possible to make phase oscillators, i.e. to drive colloids cyclically in such a way that the phase of each one is not set externally. In geometric switch models, the update of the laser trap positions is decided based on the instantaneous configuration of the particles; in stress-controlled orbits, the laser trap "driving" each colloid is maintained a predefined distance ahead of the colloidal particle, on a predefined track.

Within each class of model (configuration- or stress-controlled), many biologically and medically important elements can be tested: the role of fluid rheology, the vicinity to solid surface boundaries, the robustness to external flows and perturbations in the flow, the effects of heterogeneity in cilia spatial distribution, and the robustness to loss of cilia in patches.

\subsection{Geometric switch: The "rower" model}

4.2.1. The model. In the experiments of (77), two beads are confined in separate harmonic wells (Figure 4(a)). The position of each well is linked to the spatial configuration of the beads via a "geometric switch". Specifically, the laser trap on each particle is moved between two positions a distance apart, as shown in Figure 4(b), following the rule that the switch of trap position is triggered when a particle approaches to within a distance from the minimum of the active potential. This feedback-controlled motion of the traps is sufficient to induce sustained oscillations, and each particle undergoes long-time periodic motion with a fixed amplitude. Crucially, when more than one bead is present in the system, the geometric switch is determined independently for each bead (200 times per second in the current setup), so that the external trap forces do not themselves impose the phase of oscillation nor its period. Since the bead radius is typically a few micrometers and the trap stiffness is between 1 and $100 \mathrm{pN} / \mu \mathrm{m}$, with the typical viscosity of $1 \mathrm{mPa} \cdot \mathrm{s}$ or above, in the absence of other external forces, a particle in a harmonic potential undergoes overdamped 

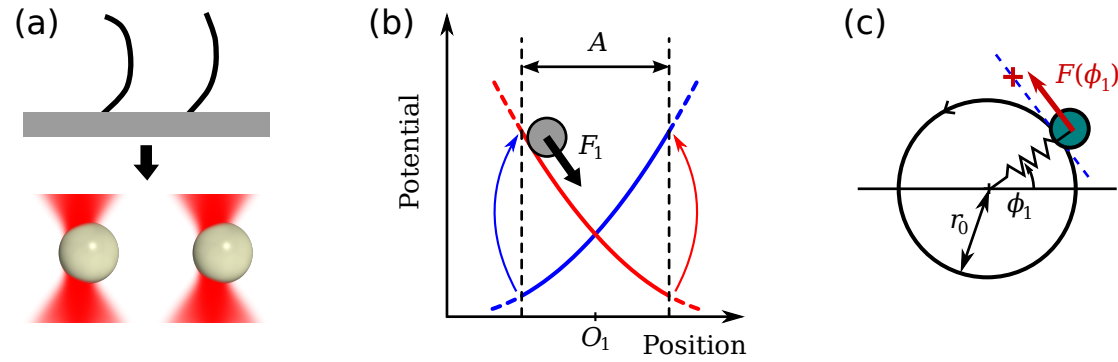

Figure 4

Colloidal model oscillators. (a) Our experimental approach to the cilia synchronization problem: Each cilium is represented by a spherical micron-sized particle, driven by optical tweezers in a way that represents the cilium beat and contains cilia properties in a coarse-grained fashion. Two classes of models have been studied extensively in theory and experiment, running multiple coupled phase-free oscillators to study their hydrodynamic synchronization. (b) Geometric switch "rower" oscillator: Two traps are defined (red and blue potentials) and are switched on and off in turn. When the trap on the right is on, the bead moves to the right, and the traps are switched when the particle reaches the position indicated by the right dashed line. It is then pushed to the left, and a second geometric boundary (left dashed line) defines the next trap switch. (c) "Rotor" model: A path is chosen for the particle (circular here). The colloid is driven by a trap that is maintained at a controlled distance $\epsilon\left(\phi_{1}\right)$ ahead of the particle, and on the tangent to the circle at the bead. The tangent force $F\left(\phi_{1}\right)$ is therefore controlled; the phase $\phi_{1}$ is determined from the bead position. In addition, the bead may deviate from the circular path in the radial direction, and a harmonic restoring force of controlled stiffness (represented by a spring on the sketch) tends to keep the particle on the predefined path.

stochastic motion driven by thermal forces (92). We more recently explored variations of this model such as two-state oscillators driven by non-harmonic potentials (19, 20).

4.2.2. Synchronization of rowers. In our first experiments (77), we showed that two driven oscillating colloidal spheres in harmonic potentials lock into antiphase motion, exhibiting a surprising behavior caused by the interplay of thermal noise and hydrodynamic interactions, as well as general features typical of coupled nonlinear oscillators such as Arnold tongues.

The shape of the driving potential also affects the state of synchronization. Wollin and Stark showed this in the geometry of a linear chain of two or more oscillators, studied numerically in the absence of noise (135). For two driven particles, we found experimentally that the curvature of the potential does indeed determine the stable synchronized state: in-phase or in antiphase (20). More specifically, harmonic-like potentials with a force that decreases when approaching the center of the well lead to antiphase synchronization, while an increasing force generates in phase synchronization. This result is illustrated by Figure 5(a). Here, the parameter $c$ characterizes the "curvature of the potential" — with constant driving forces corresponding to $c=0-$ and $\langle Q\rangle$ is an order parameter that takes a value of -1 for in-phase synchronization and +1 for antiphase motion. These experiments show a transition of the synchronized state, and were backed up by BD simulations with the coupling described by the Oseen tensor. At $c \approx 0$ (non-curved, linear potentials), the two rowers do not synchronize even at low temperature, which is a manifestation of the fact that breaking time-reversal symmetry is a necessary condition for the system to synchronize. Analytical considerations are also possible at this level of description: a theory based on the solving of the equations of motion in the presence of thermal fluctuations (the 
thermal noise leads to distributions of the particle first-passage times that trigger a trap move) successfully approximates the mean order parameter.

The role of noise in these systems is a relevant question given the micrometric size of the system (Brownian motion) and presence also of bio/chemical noise due to the molecular processes in the living systems. We have highlighted that thermal noise can prevent synchronization $(19,20)$ and trigger phase slips when the two oscillators have different intrinsic frequencies (19, 77).

The position of the "model cilia" is also very important: we looked experimentally at arrays of $N=3,4$ and 5 oscillators (and more general systems, numerically) (26, [30), making the surprising discovery that while polygonal arrays of 4 or more colloids behave like the 2-particle system, and synchronize with the nearest neighbors in antiphase (for harmonic drive), a system of 3 equally spaced colloids synchronizes in-phase. Other oddnumber systems share the property of having phase-locked travelling waves clockwise and anticlockwise. Studying these small networks, we realized that the non-equilibrium dynamical steady-state is predominantly formed by the eigenmode with longest relaxation time when the driving potentials have positive curvature (e.g. harmonic wells with a positive coefficient). On the contrary, the eigenmodes with the shortest relaxation times dominate the dynamics in systems where the curvature is negative, because modes are growing and the shortest time constant modes grow the fastest. Therefore, for a given form of the coupling tensor, the character of the collective dynamics can be predicted from the spatial configuration, and the knowledge of the type of drive. This predictive power is remarkable, since the eigenmode structure is just an equilibrium property of the passive system. The argument based on eigenmodes has been verified in non-polygonal configurations as well, although we managed to find some exceptions to this rule (85). Refs. (26, 85) also present the equations of coupling of the system in terms of "reduced" Oseen tensors that take into account that the direction of each rower is constrained, so that a relation between the $x$ and $y$ motion of a particle allows to reduce the dimension of the coupling matrix from $2 N \times 2 N$ to $N \times N$.

The Oseen eigenmodes for a given geometrical arrangement are therefore predictive of the collective motion in the active state: this is a very powerful result, useful in designing optimal geometrical arrangements for sustained collective fluid transport by these active oscillators. In arrangements of many oscillators, the coupling is strongest the closer they are geometrically. Therefore in a disordered and large system it should be possible to identify clusters, i.e. groups of 2, 3, 4 or 5 spatially close oscillators which are more tightly coupled together, as in Figure 3(b) (inset). These will synchronize into "dynamical motifs" similar to the ones we have observed in (30), perhaps enabling the larger scale (tissue level) metachronal wave to be understood as resulting from local units. It will be fascinating in the future to discover if biology makes use of this hierarchical structure, and to what level of disorder can be tolerated in a ciliated tissue. Long range metachronal waves have also been reported numerically and analytically in chains of rowers (29, 135). It was shown in (135) that metachronal waves emerged when the range of the hydrodynamic interaction was short, for example considering only the interaction between nearest neighbors, as happens in the limit of being close to a no-slip boundary. 


\subsection{Oscillators moving along orbits: The "rotor" model}

Another very useful model system is to consider stress-controlled oscillators. Here, the key control parameter is force, rather than position. This type of model is one step closer to the situation in biological cilia, where the motors inside cilia also play the role of sensors for the state of stress on the filament, although perhaps the "geometric switch" discontinuity is a good representation of a collective stress-driven detachment of motors from filaments, switching from power to recovery stroke.

4.3.1. The model. When a cilium beats, its center of drag moves along a given orbit that can be a $2 \mathrm{~d}$ or $3 \mathrm{~d}$ path. A way to model a cilium is hence to prescribe an orbit for the particle (99, 127, 128). With $\phi$ the phase of the oscillator, the orbit is described by its shape $\mathbf{r}(\phi)$ and the driving force acting on the particle $F(\phi)$, that represents the force provided by the cilium, in the direction tangent to the path at $\phi$, as shown on Figure 4(c) for circular orbits. Additionally, the orbit can be made flexible (99), which helps to break the time-reversal symmetry required for synchronization. In order to generate a net flow, the trajectory can be tilted and positioned close to a surface, so that the drag coefficient changes during the cycle because of the variable height from the wall. This idea of the possible importance of wall effects in the flows generated by cilia and flagella was already emphasized by Blake (무).

4.3.2. Understanding synchronization in rotors: role of force profile. In (128), Vilfan and Jülicher showed with numerical simulations that two beads driven on elliptical and tilted trajectories near a wall can synchronize, with their state controlled by the relative position of the orbits. In this case, synchronization comes from the hydrodynamic coupling between the two oscillators that allows the particle to move faster or slower on its trajectory than if it was just pulled by its driving force. This way of synchronization was later addressed by Uchida and Golestanian, who derived generic conditions for synchronization (126, 127). Here, a trajectory is simply defined by $\mathbf{r}(\phi)$ and $F(\phi)$ that are both functions of a phase $\phi$ and no flexibility of the orbit is assumed. The hydrodynamic coupling is described by a "geometric factor" $H_{12}\left(\phi_{1}, \phi_{2}\right)$ which is the hydrodynamic tensor projected along the tangents to the two trajectories at $\phi_{1}$ and $\phi_{2}$, where 1,2 designate the two oscillators. By linear stability analysis, they obtained that the condition for synchronization of the two oscillators is that the growth rate

$$
\Gamma \approx-\frac{2}{T_{0}} \int_{0}^{2 \pi} \mathrm{d} \phi[\ln F(\phi)]^{\prime} H_{12}(\phi, \phi)
$$

is negative (127), where $T_{0}$ is the intrinsic period of the oscillators. This formula highlights that both the driving force and $H_{12}(\phi, \phi)$ must depend on $\phi$ to obtain synchronization. Since for most trajectories the second condition is satisfied (127), the condition usually reduces to having a non-constant $F(\phi)$. The formula also gives the intuition that near-field effects can be used to synchronize the system by having strong variations of the geometric factor. By pushing the calculation further, they also defined an effective potential of synchronization $V\left(\Phi_{1}-\Phi_{2}\right)$ that allows to quickly identify the synchronized states (minima of this potential) and the likeliness of phase slips in the presence of noise (they depend on the amplitude of the potential and its possible tilt). Here $\Phi_{i}$ is a gauge linked to $\phi_{i}$, such that $\Phi_{i}$ increases linearly if the oscillator is uncoupled.

In the case of two circular trajectories (as in Figure 4(c) but without flexibility) Uchida 


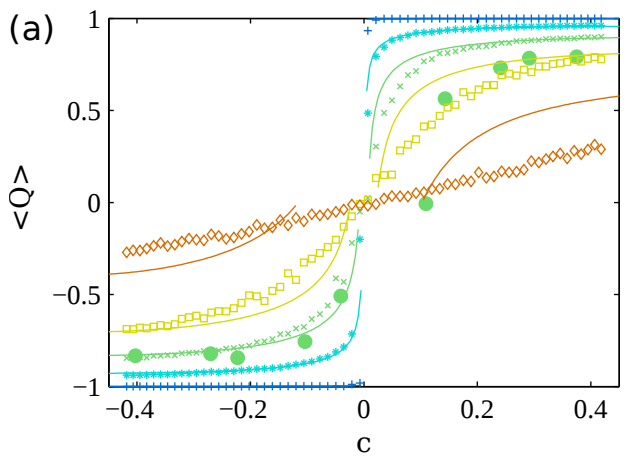

(c) $\mathrm{i}$
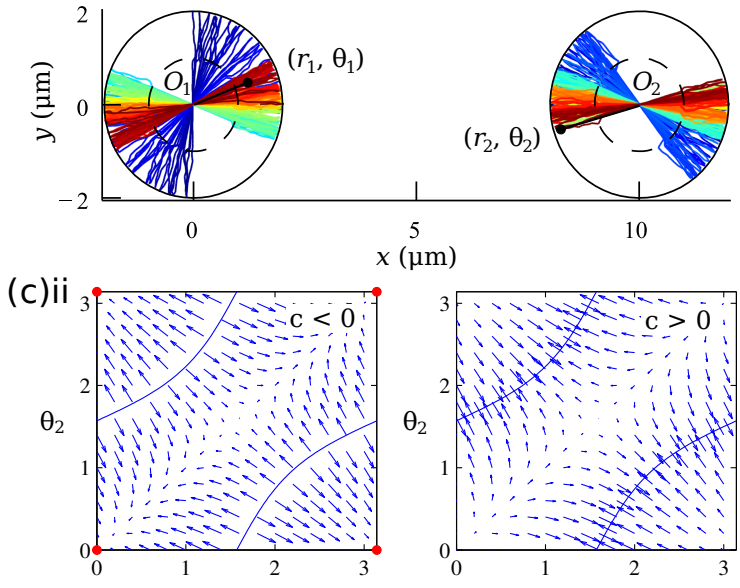

$\theta_{1}$

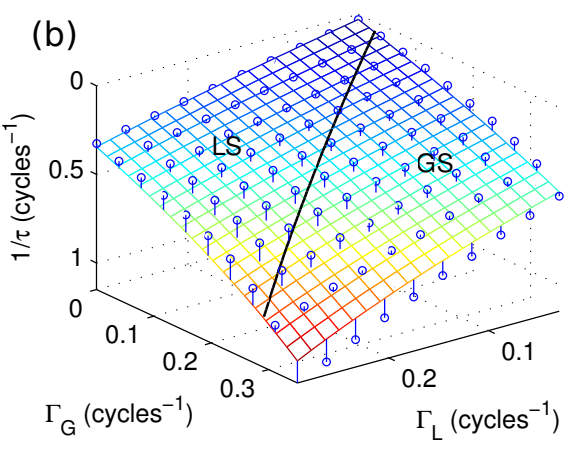

(d)

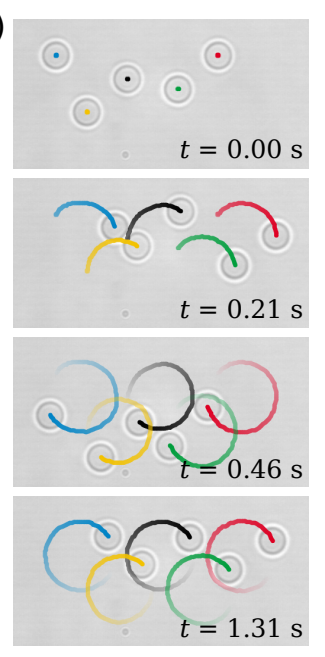

Figure 5

Main results on colloidal models. (a) Synchronization of two rowers. Depending on the curvature $c$ of the driving forces, two rowers synchronize in phase or in antiphase $(\langle Q\rangle=-1$ or 1 respectively) in experiments (filled circles), BD simulations (open markers) and theory (solid lines). The different colors indicate different temperatures (increasing from blue to red), and higher temperatures require a stronger curvature of the driving forces to see synchronization. (b) Synchronization (in phase) of two rotors: simulations (markers) and theory (colored surface). The inverse relaxation time of the phase difference $1 / \tau$ is a measure of the strength of synchronization. Here, the strength of synchronization is controlled by two dimensionless parameters $\Gamma_{\mathrm{L}}$ and $\Gamma_{\mathrm{G}}$ for the Lenz and Golestanian mechanisms, respectively. The mechanism that dominates is indicated by the LS and GS areas (Lenz and Golestanian synchronization). (c) Alignment of two rowers with a free direction of beat. (c)i Tracks of two beads in the case of negatively-curved potentials from a random initial condition (increasing time from blue to red). Each oscillator is a rower with a free direction of oscillation. Here, the beads oscillate along diameters of a circle and slowly align horizontally, i.e. $\left(\theta_{1}, \theta_{2}\right)$ tends to $(0,0)$. (c)ii Analytical convergence map of the $\left(\theta_{1}, \theta_{2}\right)$ orientation of the beats depending on the curvature. For $c<0$, the convergence to $(0,0)$ (red dots) is predicted, while for $c>0$, the pair of angles converges to a configuration on the solid lines. In the presence of Brownian noise, this system does not align, but stays close to the solid lines. (d) 5-rotor configuration that synchronizes after a few cycles. To make synchronization possible, the driving force on the central rotor needed to be reduced, as it is more coupled than others to the rest of the system. 
and Golestanian showed that in the Fourier decomposition of the force profile, the mode that leads to strongest synchronization is (127)

$$
F(\phi)=F_{0}\left[1-A_{2} \sin (2 \phi)\right]
$$

with $0<A_{2}<1$. This is valid in the far-field limit, when the size of the orbits is much smaller than the distance $d$ between the oscillators, and when the oscillators are either very close to the surface ( $h \ll d$, with $h$ the height from the surface) or in the bulk $(h \gg d)$. For these circular paths, if $A_{2}=0$, the amplitude of the driving force is constant and the system does not synchronize.

More recently, this model was used by Golestanian and Bennett to study the synchronization of Chlamydomonas in simulations: Two colloidal rotors were maintained close to a third sphere that is modelling the cell body. In the presence of noise, this lead to a run-and-tumble behavior ( $(5)$, similar to the actual alga. The synchronized states and their stability have also been studied without noise, depending on the choice of the driving force profile (므).

4.3.3. Understanding synchronization in rotors: role of trajectory compliance. As just explained, synchronization can arise from particular choices of the force profiles driving rotors. However, synchronization in rotors can also emerge from another mechanism: the flexibility (compliance) of the orbits. Flexibility leads to loss of time-reversal symmetry, and is a common way to force hydrodynamic synchronization in various systems such as rotating helices and paddles (109, 111, 112). It has been proposed for colloidal rotors by Niedermayer, Eckhardt and Lenz in (99).

This model is shown in Figure 4(c), where the orbits are circular and $F\left(\phi_{i}\right)$ is now set to a constant. Flexibility is allowed in the direction orthogonal to the orbit, meaning that instead of following exactly the circular trajectory, the particles can deviate from their orbits, for example because of the coupling with other particles or thermal fluctuations. To stay close to the predefined path, a restoring force is added which tends to pull the particle back to the track. The restoring force can, to the lowest order, be described by a spring constant as shown in Figure 4(c). For two rotors driven by constant forces and without thermal fluctuations, Niedermayer, Eckhardt and Lenz calculated the state of synchronization and its strength. They found that the decay rate of the phase, as it converges to the synchronized state (in phase), is proportional to the inverse of the spring constant $k_{r}$ that constrains the beads to the circles. In long chains of oscillators, their simulations also led to metachronal waves in both cases of periodic and free boundary conditions at the ends of the chain.

4.3.4. Towards more elaborate rotor models. The early rotor models represented above were highly simplified as they either assumed stiff orbits ("Golestanian model") or constant driving forces ("Lenz model"). These idealized views have been very useful to determine the origin of synchronization. However, a more realistic representation of a cilium should include both non-constant driving forces to represent well the power and recovery strokes of a cilium, and flexibility. This has been done experimentally with optical tweezers in (76). In Figure 4(c), for the oscillator $i$, the center of a trap is maintained ahead of the particle at a distance $\epsilon\left(\phi_{i}\right)$. The trap is controlled by a feedback loop that ensures that it is positioned, for every feedback cycle, at $\epsilon\left(\phi_{i}\right)$ along the tangent to the preprogrammed orbit, at the point at $\phi_{i}$ at which the bead is. Controlling the trap-to-bead distance sets the driving force $F\left(\phi_{i}\right)=k \epsilon\left(\phi_{i}\right)$, where $k$ is the trapping constant of the optical tweezers. Some 
degree of flexibility in the trajectory is always present when using optical tweezers. If the optical traps have the same stiffness in all directions of the focal plane, the flexibility would be simply $k$. Thus Golestanian and Lenz synchronization can be tuned independently by changing $k$ and $\epsilon\left(\phi_{i}\right)$. In practice, we have implemented complex trapping landscapes by time-sharing a laser beam with acousto-optic deflectors (see (20) for details on this method). This allowed us to study oscillators with much higher flexibility than what we would have obtained with simple harmonic traps. With this setup, we have been able to observe both Lenz and Golestanian in-phase synchronization in the configuration shown in Figure 4(c) with the force profile in Equation 10. In an intuitive analysis, we have successfully recovered the synchronization strength $\Gamma$ (i.e. the cycle-averaged decay rate of the relative phase difference at the synchronized state) as a simple sum of the Golestanian and Lenz strengths of synchronization $\Gamma_{G}$ and $\Gamma_{L}$ of the two models described above:

$$
\Gamma \approx \Gamma_{G}+\Gamma_{L}=2 \pi \frac{3 a}{4 d}\left(A_{2}+\frac{3 F_{0}}{k r} \sqrt{1-A_{2}^{2}}\right)
$$

with $a$ and $r$ the particle and orbit radii, and $d$ the distance between the oscillators. Figure 5 (b) shows a comparison between the measured inverse relaxation time $1 / \tau$ of the phase difference in simulations (circles) and the expected rate $1 / \tau \approx-\ln \left[1-\left(\Gamma_{L}+\Gamma_{G}\right)\right]$.

\subsection{Alignment of the beating planes: The "rower" with a free direction}

Synchronization of the beads is not the only cooperative behavior that coupled oscillators can display. For instance, in a carpet of cilia showing a metachronal wave, all the cilia roughly beat in the same direction. The direction of cilia beats in a fully grown epithelial tissue is well defined relative to the organ. This is essential for the generation of fluid flow, for example for mucus clearance away from the lungs, which relies on coordinated beating of cilia to produce transport-efficient metachronal waves (21). However, the origin of this orientation that develops during the tissue growth (after planar cell polarity is established) is still an open question in developmental biology. For example, the network of microtubules connecting the basal bodies could couple to the cell shape (43), and the hydrodynamic interaction may also play a role in setting the orientation (65, 66).

In the rower view of a cilium each oscillator has a direction of oscillation. While this direction was constrained in the work described in Section 4.2 we have also modified the model to allow the direction to deviate slowly because of hydrodynamic forces (details of this extended model can be found in (18) $)$.

The rower model with free orientation produces rich results. We saw that two oscillators synchronize in phase and align in the direction defined by the line joining the centers of the oscillators, if they are driven by power-law potentials with an exponent $\alpha<1(c<0$, see Figure 5(c)i). The figure shows the tracks of the two oscillators starting from an initial condition (dark blue) with a random phase and a random orientation. After a few tens of oscillations, the system converged to parallel oscillators. The in-phase synchronization (not clearly visible on the figure) is consistent with results on rowers with fixed orientation, where in-phase locked states were obtained for negative curvatures $c$ (Figure 5(a)i). For $\alpha>1$ $(c>0)$, the system does not converge to a fixed orientation: it rather chooses orientations for which the hydrodynamic coupling vanishes, and as a consequence synchronization becomes weak. The convergence of the orientations are summarised in Figure 5(c)ii, where the arrows show the map of the time evolution of the orientations $\theta_{1}$ and $\theta_{2}$ of the two oscillators. We also simulated configurations of up to 64 oscillators with a free orientation, and systems 
with $\alpha<1$ always aligned strongly, even with noise, in the direction of the elongation of the array of oscillators. This could be relevant to explain the alignment of cilia in elongated cells such as Paramecium, and perhaps in planar cell polarized tissues, see Section 6 .

\subsection{Limitations of the rower and rotor models}

An important limitation of the colloidal models is that it is hard to implement experimentally colloidal oscillators in a way that they generate a net fluid flow, since typically a spherical bead is undergoing a back-and-forth motion. Swimmers or micropumps in which the traps are switched with a known frequency (hence no free phase oscillators), were however realized experimentally by Cicuta et al. (82, 83) with two or three beads, based on model swimmers proposed in (38, 58, 59, 98). In an extensive study, Pande and Smith showed that synchronization between the arms of a three-bead swimmer can maximize its net velocity (103). A model oscillator that generates a net flow should have many degrees of freedom, and good candidates for a model may be self-assembled magnetic colloids (129, 130).

The current challenge is to develop experimental colloid models that account for more realistic biological conditions. Some steps towards that have been made with the rower with a free direction of oscillation, or with the circular rotor model that includes both a non-constant driving force and flexibility. An extension of the rotor model could investigate the case of non-circular trajectories experimentally. The motion of the center of mass of a Chlamydomonas flagellum is indeed far from circular (see supplementary material of (20)). More importantly, the effect of the surface on which the cilia are anchored is often neglected. The main effect of the surface is to shorten the range of the hydrodynamic coupling, from a $1 / d$ to a $1 / d^{3}$ decay between a bulk fluid and close to the surface. While this is not very relevant when studying only two oscillators if the typical size of the oscillations is small compared to the distance between oscillators, as it only changes the amplitude of the coupling, it can have an effect on the collective dynamics of more than two oscillators (135). Finally, many biological fluids that surround the cilia, like mucus, are highly non-Newtonian, and in some cases not even homogeneous. This can affect both the motion of a single cilium and the collective properties of an assembly of oscillators. While experiments similar to those described above could be simply reproduced in a viscoelastic fluid, simulating the model and obtaining analytical determinations of the synchronization properties will pose entirely new challenges.

\section{BIOLOGICAL SYSTEMS AND LINKS WITH MODELS}

Particular strategies have been developed by microorganisms to enable motility in liquids. The most well studied cases are bacteria flagella (31) and the eukaryotic cell cilia, reviewed in (81). Few experiments exist on waves involving large numbers of real cilia, with resolution at or close to individual units: Okamoto and Nakaoka reconstituted cortical sheets of Paramecium ciliated cells, and observed the emergence of collective cilia beating (100), Berg and coworkers have studied "bacteria carpets" (31). The airway tissue is an "intrinsic" and bio/medically relevant system and is experimentally accessible. This section aims to overview the experimental observations that have been made on increasingly complex (larger cilia number) living systems, and the models that have been proposed to explain synchronized collective motions. 


\subsection{Single cell organisms with few cilia}

With only two flagella, Chlamydomonas has been a successful system to investigate synchronization. This organism can be easily handled experimentally, and many mutants are available to tune several parameters, like making it uniflagellar (131). Relevant information can be obtained both to gain access to the swimming cycle of the alga and to characterize the synchronization of the flagella. For example, we have used data on the cycle of beat of a flagellum (2) to determine the shape of the driving potential that can be inputted in the rower model. Using this potential lead to a prediction on the synchronization state consistent with the breaststroke motion of Chlamydomonas that dominates in the wild type of the alga (20), see Figure 1(b). Goldstein et al. followed another approach — characterizing the synchronization. They observed that the swimming of the alga also includes short periods of phase drifts during which the flagella oscillate asynchronously (55, 106). This is linked to the organism's motility: Chlamydomonas displays periods of long straight trajectories, alternating with abrupt changes of direction, similarly to the "run-and-tumble" motility well known in prokaryotes (106). A recent study found consistency between this behavior of the two flagella and a generic model of two coupled noisy phase oscillators with a coupling strength consistent with hydrodynamic interactions (55), and the tumble looks similar to the antiphase state seen in the ptx1 mutant (133). Hydrodynamic forces are certainly sufficient to induce synchronization of the flagella as beautifully demonstrated in (17), but there is also evidence that in the swimming state the mechanical coupling through the motion of the central cell body also needs to be considered (47).

\subsection{Metachronal waves in Paramecium and Volvox}

Paramecium and Volvox carteri are respectively unicellular and multicellular organisms, a few hundreds of micrometers in size, with an outer surface covered with thousands of cilia (75, 131): They are model organisms for imaging metachronal waves.

In the colonial alga Volvox, each of the ciliated cells composing the carpet of filaments is biflagellated. These two organisms show an interesting collective motion, in which the flagella do not all beat with the same phase. Instead, two neighboring flagella will keep a constant, usually small, phase difference. When oscillating, this leads to the formation of a metachronal wave that can be seen at the surface of various cells or tissues $(1,9,63$, 100, 116, 120). In Volvox, this propagating phase pattern is also perturbed by defects (local phase shifts that appear and disappear periodically) and this dynamics has been reproduced in a system of hydrodynamically coupled tilted rotors near a surface (15).

In-phase synchronization and metachronal waves are believed to optimize the flow generated by an assembly of cilia (94, 101).

\subsection{Mammalian mucociliary tissues}

In mammalian cilia (in the brain ventricles, fallopian tubes, airways, etc.), the cilia belong to multiciliated cells, and there are typically of order 200 cilia per cell, and each cilium is separated by approximately $200 \mathrm{~nm}$. These ciliated cells, together with mucus-producing cells (goblet cells, in mammals), and ion-regulating cells, form a general tissue type known as mucociliary epithelium. Experiments have considered developing embryos of mice (45, 119), and closely related phenomena take place on the outer surface of various "model" biological organisms, such as the Paramecium (4) and the algae colony Volvox (16) as well as and 

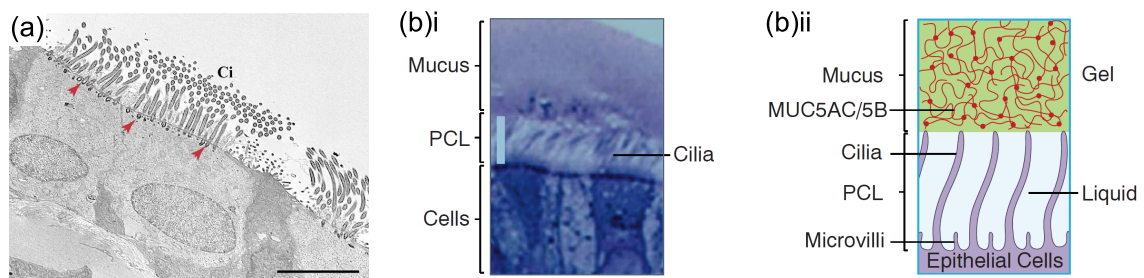

Figure 6

Side views of trachea epithelial cilia. (a) Mature ciliated cells in rat trachea (32). During ciliogenesis, centrioles develop into basal bodies (red arrows). These have extended cilia (scale bar: $5 \mu \mathrm{m}$ ). (b) Periciliary layer (PCL) (21). The gel-like mucus phase "sits" on the cilia brush without extending in the cilia layer. Instead of mucus, the cilia are surrounded by a liquid phase, the PCL (scale bar: $7 \mu \mathrm{m}$ ).

\section{Xenopus frogs (37).}

Apart from understanding their synchronization and cooperative behavior, ciliated tissues raise other interesting questions: for example, in multiciliated cell tissues (carpets of many cilia) the cilia all beat in parallel planes, posing the question of how the direction is chosen in tissue development (43) and maintenance. Cilia grow outwards from a structure called the basal body, which is anchored to a cell's cytoskeleton (97) (Figure 6(a)). The basal body is itself generated from centrioles, which are subcellular structures responsible for microtubular organisation. Once fully grown, a cilium transverses the cell's plasma membrane extending typically several microns out of the cell body. In fully developed epithelial tissue, the direction of beating is well defined relative to the organ, for example it is parallel to the trachea in mammalian airways. This is essential for the generation of fluid flow (mucus clearance away from the lungs in this case) which relies on coordinated beating of cilia to produce transport-efficient metachronal waves (21), similarly to Paramecium and Volvox.

\subsection{Respiratory tract}

Particularly important in terms of human health, and impact of related disease, is the respiratory tract. The mucociliary clearance process is the first line of defence against a wide range of potentially harmful agents including bacteria, viruses, and particulate matter. The layer of mucus (ions, water, mucin proteins), kept in motion by beating cilia and occasionally by cough, acts as a physical barrier. Flow of fluid is maintained over all the airway surfaces, toward the larynx. As reviewed in (88), dysfunction in this clearing system, which can arise from conditions such as human cystic fibrosis (CF) and primary ciliary dyskinesia (PCD), has severe consequences such as chronic lung infections and respiratory insufficiency. Altered mucus clearance is also linked to other chronic airway diseases such as asthma that afflict millions worldwide.

5.4.1. Mucus clearance by cilia. The human bronchial system is complex: epithelium is mucociliated and pseudo-stratified in vivo, and the mechanisms that regulate its functions are not fully understood. Among the epithelial cells, goblet cells secrete mucus, which protects the bronchial epithelium and is transported by the motile cilia protruding from the apical poles of specialized epithelial cells (Figure 6(a)). The coordinated system of 
epithelial cells — including functions as ion transport through their membranes, mucus secretions and cilia action - and cough, is collectively termed mucus clearance, and results in a continuous flow. The respiratory tract is lined by a thin layer of airway surface liquid, which is approximately 7-70 $\mu \mathrm{m}$ in height (21) (Figure 6.(b)). This layer is itself defined as consisting of two phases: an outer mucus-rich layer, and an inner fluid close to the cilia, known as "periciliary liquid layer" (PCL). The PCL height is in practice defined as height of the extended cilium, and recent studies have demonstrated complex interactions among cilia, the mobile mucins in the mucus layer, and the underlying PCL (122). It appears that both the mucus layer and the PCL are moved unidirectionally, and it is suggested that the movement of the airway surface liquid (ASL) involves two steps (21): First, the ciliary power stroke acts on the under-surface of the mucus to move the mucus layer unidirectionally on the airway surface; second, the frictional interaction of the mucus layer with the PCL allows this underlying layer to travel at velocities similar to those of the overlying mucus layer (a form of "secondary" transport). These data are consistent with the fact that mammalian airway cilia are too short, and their recovery stroke not sufficiently close to the cell surface, to move the PCL directly. The flow within the boundary PCL layer itself is very complex, and has been addressed from a colloidal model perspective by (130).

5.4.2. Analysis of collective motion on airway cells. Various types of experiments are possible relating to airway cells: (a) sections of the trachea can be taken from animals, and maintained in a living functioning state for over a day; (b) human cells can be obtained by non-invasive procedures (nose brush or scrape) and cultured to recreate an in vitro tissue; (c) ciliated cell lines, and fully confluent tissues, are available commercially. Cells in all these in vitro systems express their normal mucus layer (which can be temporarily washed-off by buffer). The choice of experimental approach depends on the question of interest.

The physical properties of mucus, including rheological parameters, are important in setting the frequency of cilia beating and they have been investigated extensively in the context of various pathologies (123). For example, in cystic fibrosis there is clear evidence that the properties of the PCL are abnormal. Existing observations in the literature of ciliated tissues have focused mainly on measuring cilia beat frequency and mean flow velocity.

\section{OUTLOOK}

A key objective is to link quantitatively the emergent collective dynamics to the behavior of the individual oscillators, and to the physical properties of the materials surrounding the cilia. Current models are minimal, and realistic aspects of the real systems will need to be examined and included into the models, such as viscoelasticity of the liquid, the presence of nearby surfaces (130), heterogeneity of cilia distribution (125), plasticity of beat orientation. Linking all the scales of this problem, i.e. how collective dynamical phenotypes emerge from the molecular functioning of the cilium, remains a formidable long-term challenge, requiring combined experiments on colloidal models, simple biological organisms, and complex biological tissues. Understanding this link will be a breakthrough in the physics of synchronization and will open up diagnostic potential for example in the context of pathologies of the airway tract. 


\subsection{Clinical diagnosis of diseases}

Clinical diagnosis most commonly consists of inspection of cilia beat frequency, and cilia waveform, from small biopsies. In cases of suspected genetic disease (PCD), the ciliar molecular structure is assessed by electron microscopy. Key aspects of cilia dynamics (such as space and time coherence) are currently not being addressed in the clinical setting, but they are evidently important in conditions leading to "patchy loss" of cilia, which can be a consequence of explosion trauma or infections. They will also be key in understanding the efficacy of drugs used to treat symptoms of CF or asthma, and in future regenerative medicine to aid development of functional tissues and define standards. The role of fundamental physics in this context is to highlight which dynamical patterns and which parameters of the driving force are important, so that these significant parameters can be investigated and measured in the biological system.

One challenge is to go beyond this analysis, and to extract more information, which would feed into a two-way link between the collective steady state and the function at the single cilium level. In this way, from easily measurable macroscopic behaviors, one would be able to infer microscopic properties. For example, recent developments push towards resolving the shape of a single cilium during the power and recovery strokes, which is now possible in simple organisms (2). These experiments are important in relating to the microscopic molecular function within a cilium. If one had high resolution observations of the shape of a beating cilium during each stroke, and knowledge of the mechanical properties of the filament bundle, this could be linked quantitatively with models that account for cilia structure and molecular motor activity such as (70). However another question remains, which is at least as important because it too offers a (possibly experimentally easier) way to link the macroscopic to the molecular scale: given a force versus time profile throughout the stroke (which can be thought as an intrinsic property of a certain cilium in given external physical conditions), what is the resulting collective property of the tissue surface, in which many such cilia are spatially arranged?

Answering this challenge requires bringing together fundamental ideas and carrying out clear experiments on the biological systems to prove, control and demonstrate the relevance of what is identified from the colloid model experiments. A clear understanding of how the bottom-up emergence of certain dynamical motifs (from given force profiles, geometrical arrangements, and coupling forces) is achieved, will provide a powerful tool: a map that makes it possible to relate these motifs back to those microscopic properties, which could then feed into a video analysis package that could be deployed in the clinical setting for the study of pathologies linked to ciliary dysfunction.

\subsection{Development of ciliated tissues}

An open question in developmental biology is to find the rules and the cues that enable cilia tissues, a fairly complex, and well organized carpet, to be made, and in particular, how the direction of the cilia is chosen. The first symmetry to be broken in the development of vertebrates is the anterior/posterior. For example the planar cell polarity (PCP) pathway sets the initial direction in a developmental stage of the epithelium in Xenopus embryos, a tissue which includes multiciliated cells (96). From this point on, there are gradients of a variety of biochemical elements along this axis. Cells can be polarized, both in the intracellular protein localization, and in their shape. This process happens before ciliagenesis, and the standard view in biology is that the gradients in biochemical markers control 
most, if not all, of the subsequent organ development. However, once cilia are generated, they contribute to long-range flows, which can transport chemical factors directionally, or act as a mechanical cue for organization (46, 91, 95, 132).

In the specific case of developing orientational cilia order in the airway tissue, there is a hypothesis that flow-induced self organisation might be important. A fraction of the cells in the tissue that will develop into the airway epithelium express a few hundred centrioles, which become basal bodies and grow cilia. At this stage, the cells themselves are already polarized (biochemically and in shape) but the basal bodies when they first appear are not oriented. There have been very recent studies suggesting that the network of microtubules connecting the basal bodies could couple to the cell shape or to the emerging tissue architecture, and possibly orient the cilia (43). On the other hand, the newly made cilia are exposed to a directional flow from the mucus being produced by other cells. These cilia will also be exposed to the flow that they themselves generate, i.e. they interact with each other through hydrodynamic interaction forces $(\underline{63})$. The question of how are cilia aligned has also been addressed looking at mouse brain ventricles (66), where it was shown that cilia first dock apically with random orientations, and then reorient in a common direction through a coupling between hydrodynamic forces and the PCP protein Vangl2.

The evidence of the role of flows in determining the orientation of cilia is therefore present in the experiments in the Xenopus larval skin (95, 134) and in mouse brain ventricles (66). However, there are only very few physical models to explain this behavior (65). Experiments where air or liquids are driven across the cell laden surfaces will provide insight as to whether and at what development stages these stresses can reorient cilia attachment and resulting active transport.

\subsection{Basic science and microfluidic technology}

The models discussed here, showing synchronization regimes that are tunable, and competing with Brownian noise, are also very accessible platforms to develop the science of nonlinear systems (105). In this, they have the "usual" great beauty in common with many colloidal particle model systems: Easily accessible timescales, direct imaging of configurations, intrinsic presence of stochastic elements (through thermal noise). Our understanding of which configurations, geometries and coupling forms might be ideal to maximize susceptibility, or create active media that support particular signal propagations, is still very crude.

In the technological arena, taming appropriately the mechanisms of hydrodynamic synchronization could make it possible to optimize devices such as micro-pumps (74), molecular sorters and low-Reynolds number mixers, and control behavior of swarms of swimmers. Various groups have succeeded in generating artificial carpets of beating filaments (52), but not yet with free phase. If such systems had spontaneous synchronization, this might be designed in such a way that it can sustain fluid drive over a variety of conditions (as do the biological mucociliary tissues), whereas a deterministically driven carpet is necessarily tuned for just one condition.

\section{SUMMARY POINTS}

1. Model systems show that hydrodynamic forces, exerted by one cilium on another, can be sufficient to cause synchronization, and emergence of collective wave states. 
2. The properties of the emergent collective behavior depend on a number of factors, which can be individually addressed (experimentally, numerically and analytically) working with simple models, in which each motile cilium is coarse grained into the movement of a single colloidal sphere, retaining elements of the original system in the details of the drive and geometric disposition.

3. Two main classes of models (rowers and rotors) have been studied so far; both can be mapped onto biological data, both have rich phenomenology, and each has particular aspects that make the model attractive.

4. Model systems have been used to highlight the regime of synchronization, which is limited by thermal noise, and by detuning of the natural frequencies of the driven oscillators.

5. Model systems are also able to explain the type of synchronization; the main thrust of work so far has revolved around mapping the system of the biflagellated algae Chlamydomonas, and addressing how it manages to exhibit both in-phase and antiphase beating patterns.

\section{FUTURE ISSUES}

1. Our fundamental understanding of this class of non-linear systems is still incomplete, and the aim of linking local rules to the macroscopic properties of emergent dynamics is in general still an open challenge.

2. The class of non-linear systems described here has so far been explored as models of biological motile cilia, but there are many other future areas of development in technology (artificial surfaces that drive fluid flow) and micro-scale sensing (optimized arrays for picking up faint signals).

3. Understanding robustness against geometric heterogeneity: In ciliated tissues, there are clusters of nearby cilia (e.g. from the same cell), and longer distances in between ciliated cells; in some diseases, patchy loss of cilia leads to regions devoid of beating cilia.

4. Hoping to describe ciliated tissues such as the airways, it will be necessary to understand the role not just of mucus viscoelasticity, but also of its complex stratified nature.

5. This area of work is partially justified by clinical challenges, and aims to improve diagnostics and perhaps even therapeutic practice relating to conditions where mucociliary clearance is compromised.

\section{DISCLOSURE STATEMENT}

The authors are not aware of any affiliations, memberships, funding, or financial holdings that might be perceived as affecting the objectivity of this review. 


\section{ACKNOWLEDGMENTS}

We thank all of our collaborators working in this area, particularly M. Cosentino Lagomarsino for introducing us to this question many years ago, and many discussions since then, and L. Feriani and M. Chioccioli for providing the images in Figures 1(c)i and 3(b). This work is funded by the ERC Consolidator Grant HydroSync.

\section{LITERATURE CITED}

1. Aiello E, Sleigh MA. 1972. The metachronal wave of lateral cilia of Mytilus edulis. J. Cell Biol. 54:493-506

2. Bayly PV, Lewis BL, Kemp PS, Pless RB, Dutcher SK. 2010. Efficient spatiotemporal analysis of the flagellar waveform of chlamydomonas reinhardtii. Cytoskeleton 67:56-69

3. Beatus T, Tlusty T, Bar-Ziv R. 2006. Phonons in a one-dimensional microfluidic crystal. Nature Phys. 2:743-748

4. Beisson J, Jerka-Dziadosz M. 1999. Polarities of the centriolar structure: Morphogenetic consequences. Biol. Cell 91:367-378

5. Bennett RR, Golestanian R. 2013. Emergent run-and-tumble behavior in a simple model of Chlamydomonas with intrinsic noise. Phys. Rev. Lett. 110:148102

6. Bennett RR, Golestanian R. 2013. Phase-dependent forcing and synchronization in the threesphere model of chlamydomonas. New Journal of Physics 15:075028

7. Blake JR. 1971. A note on the image system for a stokeslet in a no-slip boundary. Math. Proc. Camb. Phil. Soc. 70:303-310

8. Blake JR, Liron N, Aldis GK. 1982. Flow patterns around ciliated microorganisms and in ciliated ducts. J. Theor. Biol. 98:127-141

9. Blake JR, Sleigh MA. 1974. Mechanics of ciliary locomotion. Biol. Rev. 49:85-125

10. Bray D. 2000. Cell movements: From molecules to motility. New York: Garland Science

11. Breunig JJ, Arellano JI, Rakic P. 2010. Cilia in the brain: going with the flow. Nat. Neurosci. 13:654-655

12. Brokaw CJ. 2009. Simulation of cyclic dynein-driven sliding, splitting, and reassociation in an outer doublet pair. Biophys. J. 97:2939-2947

13. Brokaw CJ. 2009. Thinking about flagellar oscillation. Cell Motil. Cytoskeleton 66:425-436

14. Brooks E, Wallingford J. 2014. Multiciliated cells. Current Biol. 24:R973-R982

15. Brumley DR, Polin M, Pedley TJ, Goldstein RE. 2015. Metachronal waves in the flagellar beating of volvox and their hydrodynamic origin. Journal of The Royal Society Interface 12

16. Brumley DR, Polin M, Pedley TJ, Goldstein RE. 2015. Metachronal waves in the flagellar beating of volvox and their hydrodynamic origin. J. Roy. Soc.: Interface 12:20141358

17. Brumley DR, Wan KY, Polin M, Goldstein RE. 2014. Flagellar synchronization through direct hydrodynamic interactions. eLife 3:e02750

18. Bruot N, Cicuta P. 2013. Emergence of polar order and cooperativity in hydrodynamically coupled model cilia. J. R. Soc.: Interface 10:20130571

19. Bruot N, Damet L, Kotar J, Cicuta P, Cosentino Lagomarsino M. 2011. Noise and synchronization of a single active colloid. Phys. Rev. Lett. 107:094101

20. Bruot N, Kotar J, de Lillo F, Cosentino Lagomarsino M, Cicuta P. 2012. Driving potential and noise level determine the synchronization state of hydrodynamically coupled oscillators. Phys. Rev. Lett. 109:164103

21. Button B, Cai LH, Ehre C, Kesimer M, Hill DB, et al. 2012. A periciliary brush promotes the lung health by separating the mucus layer from airway epithelia. Science 337:937-941

22. Cartwright JHE, Piro N, Piro O, Tuval I. 2008. Fluid dynamics of nodal flow and leftright patterning in development. Develop. Dynamics 237:3477-3490

23. Chilvers MA, O'Callaghan C. 2000. Analysis of ciliary beat pattern and beat frequency using 
digital high speed imaging: comparison with the photomultiplier and photodiode methods. Thorax $55: 314-317$

24. Chwang AT, Wu TYT. 1975. Hydromechanics of low-reynolds-number flow. part 2. singularity method for stokes flows. J. Fluid Mech. 67:787-815

25. Cicuta GM, Kotar J, Brown AT, Noh J, Cicuta P. 2010. Hydrodynamic coupling in polygonal arrays of colloids: Experimental and analytical results. Phys. Rev. E 81:051403

26. Cicuta GM, Onofri E, Cosentino Lagomarsino M, Cicuta P. 2012. Patterns of synchronization in the hydrodynamic coupling of active colloids. Phys. Rev. E 85:016203

27. Coq N, Bricard A, Delapierre FD, Malaquin L, du Roure O, et al. 2011. Collective beating of artificial microcilia. Phys. Rev. Lett. 107:014501

28. Cosentino Lagomarsino M, Bassetti B, Jona P. 2002. Rowers coupled hydrodynamically. modeling possible mechanisms for the cooperation of cilia. Eur. Phys. J. B 26:81-88

29. Cosentino Lagomarsino M, Jona P, Bassetti B. 2003. Metachronal waves for deterministic switching two-state oscillators with hydrodynamic interaction. Phys. Rev. E 68:021908

30. Damet L, Cicuta GM, Kotar J, Cosentino Lagomarsino M, Cicuta P. 2012. Hydrodynamically synchronized states in active colloidal arrays. Soft Matter 8:8672

31. Darnton N, Turner L, Breuer K, Berg HC. 2004. Moving fluid with bacterial carpets. Biophysical Journal 86:1863 - 1870

32. Dawe HR, Farr H, Gull K. 2007. Centriole/basal body morphogenesis and migration during ciliogenesis in animal cells. Journal of Cell Science 120:7-15

33. Dhont JKG. 1996. An introduction to dynamics of colloids. Elsevier Science

34. Di Leonardo R, Buzas A, Kelemen L, Vizsnyiczai G, Oroszi L, Ormos P. 2012. Hydrodynamic synchronization of light driven microrotors. Phys. Rev. Lett. 109:034104

35. Doi M, Edwards SF. 1986. The theory of polymer dynamics. New York: Oxford University Press

36. Dreyfus R, Baudry J, Roper ML, Fermigier M, Stone HA, Bibette J. 2005. Microscopic artificial swimmers. Nature 437:862-865

37. Dubaissi E, Papalopulu N. 2011. Embryonic frog epidermis: a model for the study of cell-cell interactions in the development of mucociliary disease. Dis. Model. Mech. 4:179-192

38. Earl DJ, Pooley CM, Ryder JF, Bredberg I, Yeomans JM. 2007. Modeling microscopic swimmers at low reynolds number. J. Chem. Phys. 126:064703

39. Elfring GJ, Lauga E. 2009. Hydrodynamic phase locking of swimming microorganisms. Phys. Rev. Lett. 103:088101

40. Elgeti J, Gompper G. 2013. Emergence of metachronal waves in cilia arrays. Proc. Natl. Acad. Sci. 110:4470-4475

41. Elgeti J, Winkler RG, Gompper G. 2015. Physics of microswimmers - single particle motion and collective behavior: a review. Rep. Prog. Phys. 78:056601

42. Ermak DL, McCammon JA. 1978. Brownian dynamics with hydrodynamic interactions. J. Chem. Phys. 69:1352-1360

43. Eszter KV, Roy DB, Ashvin MS, Matthew PS, Jeffrey DA. 2012. Microtubules enable the planar cell polarity of airway cilia. Curr. Biol. 22:2203-2212

44. Fliegauf M, Benzing T, Omran H. 2007. When cilia go bad: cilia defects and ciliopathies. Nat Rev Mol Cell Biol 8:880-893

45. Francis RJB, Chatterjee B, Loges NT, Zentgraf H, Omran H, Lo CW. 2009. Initiation and maturation of cilia-generated flow in newborn and postnatal mouse airway. Am. J. Physiol. 296:L1067L1075

46. Freund JB, Goetz JG, Hill KL, Vermot J. 2012. Fluid flows and forces in development: functions, features and biophysical principles. Development 139:1229-1245

47. Friedrich BM, Jülicher F. 2012. Flagellar synchronization independent of hydrodynamic interactions. Phys. Rev. Lett. 109:138102

48. Fürthauer S, Ramaswamy S. 2013. Phase-synchronized state of oriented active fluids. Phys. 
Rev. Lett. 111:238102

49. Gadêlha H, Gaffney EA, Goriely A. 2013. The counterbend phenomenon in flagellar axonemes and cross-linked filament bundles. Proc. Natl. Acad. Sci. 110:12180-12185

50. Gaffney E, Gadelha H, Smith D, Blake J, Kirkman-Brown J. 2011. Mammalian sperm motility: Observation and theory. Annu. Rev. Fluid Mech. 43:501

51. Gauger E, Downton M, Stark H. 2009. Fluid transport at low reynolds number with magnetically actuated artificial cilia. Eur. Phys. J. E 28:231-242

52. Georgilas I, Adamatzky A, Barr D, Dudek P, Melhuish C. 2014. In Nature Inspired Cooperative Strategies for Optimization (NICSO 2013), eds. G Terrazas, FEB Otero, AD Masegosa, vol. 512 of Studies in Computational Intelligence. Springer International Publishing, 261-271

53. Geyer VF, Jülicher F, Howard J, Friedrich BM. 2013. Cell-body rocking is a dominant mechanism for flagellar synchronization in a swimming alga. Proceedings of the National Academy of Sciences 110:18058-18063

54. Gheber L, Priel Z. 1994. Metachronal activity of cultured mucociliary epithelium under normal and stimulated conditions. Cell Motility and the Cytoskeleton 28:333-345

55. Goldstein R, Polin M, Tuval I. 2009. Noise and synchronization in pairs of beating eukaryotic flagella. Phys. Rev. Lett. 103:168103

56. Goldstein RE. 2015. Green algae as model organisms for biological fluid dynamics. Ann. Rev. Fluid Mech. 47:343-375

57. Goldstein RE, Polin M, Tuval I. 2011. Emergence of synchronized beating during the regrowth of eukaryotic flagella. Phys. Rev. Lett. 107:148103

58. Golestanian R, Ajdari A. 2008. Analytic results for the three-sphere swimmer at low reynolds number. Phys. Rev. E 77:036308

59. Golestanian R, Ajdari A. 2008. Mechanical response of a small swimmer driven by conformational transitions. Phys. Rev. Lett. 100:038101

60. Golestanian R, Yeomans J, Uchida N. 2011. Hydrodynamic synchronization at low reynolds number. Soft Matter 7:3074

61. Gray J, Hancock GJ. 1955. The propulsion of sea-urchin spermatozoa. J. Exp. Biol. 32:802-814

62. Gueron S, Levit-Gurevich K. 1999. Energetic considerations of ciliary beating and the advantage of metachronal coordination. Proc. Natl. Acad. Sci. USA 96:12240-12245

63. Gueron S, Levit-Gurevich K, Liron N, Blum J. 1997. Cilia internal mechanism and metachronal coordination as the result of hydrodynamical coupling. Proc. Natl. Acad. Sci. 94:6001-6006

64. Gueron S, Liron N. 1992. Ciliary motion modeling, and dynamic multicilia interactions. Biophys. J. $63: 1045-1058$

65. Guirao B, Joanny JF. 2007. Spontaneous creation of macroscopic flow and metachronal waves in an array of cilia. Biophys. J. 92:1900-1917

66. Guirao B, Meunier A, Mortaud S, Aguilar A, Corsi JM, et al. 2010. Coupling between hydrodynamic forces and planar cell polarity orients mammalian motile cilia. Nat. Cell Biol. 12:341-350

67. Hänggi P, Marchesoni F. 2009. Artificial brownian motors: Controlling transport on the nanoscale. Rev. Mod. Phys. 81:387-441

68. Happel J, Brenner H. 1983. Low reynolds number hydrodynamics: with special applications to particulate media. New York: Kluwer

69. Hilfinger A, Chattopadhyay A, Jülicher F. 2009. Nonlinear dynamics of cilia and flagella. Phys. Rev. E 79:051918

70. Hilfinger A, Jülicher F. 2008. The chirality of ciliary beats. Phys. Biol. 5:016003

71. Kavre I, Vilfan A, Babič Dcv. 2015. Hydrodynamic synchronization of autonomously oscillating optically trapped particles. Phys. Rev. E 91:031002

72. Keißner A, Brücker C. 2012. Directional fluid transport along artificial ciliary surfaces with base-layer actuation of counter-rotating orbital beating patterns. Soft Matter 8:5342-5349

73. Khaderi S, den Toonder JMJ, Onck PR. 2012. Fluid flow due to collective non-reciprocal motion of symmetrically-beating artificial cilia. Biomicrofluidics 6:014106 
74. Khaderi SN, den Toonder JMJ, Onck PR. 2011. Microfluidic propulsion by the metachronal beating of magnetic artificial cilia: a numerical analysis. Journal of Fluid Mechanics 688:44-65

75. Kirk DL. 1998. Volvox: Molecular-genetic origins of multicellularity and cellular differentiation. Cambridge University Press

76. Kotar J, Debono L, Bruot N, Box S, Phillips D, et al. 2013. Optimal hydrodynamic synchronization of colloidal rotors. Phys. Rev. Lett. 111:228103

77. Kotar J, Leoni M, Bassetti B, Cosentino Lagomarsino M, Cicuta P. 2010. Hydrodynamic synchronization of colloidal oscillators. Proc. Natl. Acad. Sci. 107:7669-7673

78. Koumakis N, Di Leonardo R. 2013. Stochastic hydrodynamic synchronization in rotating energy landscapes. Phys. Rev. Lett. 110:174103

79. Kozlov AS, Baumgart J, Risler T, Versteegh CPC, Hudspeth AJ. 2011. Forces between clustered stereocilia minimize friction in the ear on a subnanometre scale. Nature 474:376-379

80. Lauga E. 2011. Life around the scallop theorem. Soft Matter 7:3060 - 3065

81. Lauga E, Powers TR. 2009. The hydrodynamics of swimming microorganisms. Rep. Prog. Phys. 72:096601

82. Leoni M, Bassetti B, Kotar J, Cicuta P, Cosentino Lagomarsino M. 2010. Minimal two-sphere model of the generation of fluid flow at low reynolds numbers. Phys. Rev. E 81:036304

83. Leoni M, Kotar J, Bassetti B, Cicuta P, Cosentino Lagomarsino M. 2009. A basic swimmer at low reynolds number. Soft Matter 5:472 - 476

84. Leopold PL, O'Mahony MJ, Lian XJ, Tilley AE, Harvey BG, Crystal RG. 2009. Smoking is associated with shortened airway cilia. PLoS ONE 4:e8157

85. Lhermerout R, Bruot N, Cicuta GM, Kotar J, Cicuta P. 2012. Collective synchronization states in arrays of driven colloidal oscillators. New J. Phys. 14:105023

86. Lighthill J. 1976. Flagellar hydrodynamics: The john von neumann lecture, 1975. SIAM Review 18:161-230

87. Lindemann C, Lesich K. 2010. Flagellar and ciliary beating: the proven and the possible. J. Cell. Sci. 123:519-28

88. Livraghi A, Randell SH. 2007. Cystic fibrosis and other respiratory diseases of impaired mucus clearance. Toxicologic Pathology 35:116-129

89. Ma R, Klindt GS, Riedel-Kruse IH, Jülicher F, Friedrich BM. 2014. Active phase and amplitude fluctuations of flagellar beating. Phys. Rev. Lett. 113:048101

90. Marchetti MC, Joanny JF, Ramaswamy S, Liverpool TB, Prost J, et al. 2013. Hydrodynamics of soft active matter. Rev. Mod. Phys. 85:1143

91. Marshall WF, Kintner C. 2008. Cilia orientation and the fluid mechanics of development. Curr. Opin. Cell Biol. 20:48-52. Cell structure and dynamics

92. Meiners JC, Quake SR. 1999. Direct measurement of hydrodynamic cross correlations between two particles in an external potential. Phys. Rev. Lett. 82:2211-2214

93. Mercer RR, Russell ML, Roggli VL, Crapo JD. 1994. Cell number and distribution in human and rat airways. Am. J. Respir. Cell. Mol. Biol. 10:613-624

94. Mettot C, Lauga E. 2011. Energetics of synchronized states in three-dimensional beating flagella. Phys. Rev. E 84:061905

95. Mitchell B, Jacobs R, Li J, Chien S, Kintner C. 2007. A positive feedback mechanism governs the polarity and motion of motile cilia. Nature 447:97-101

96. Mitchell B, Stubbs JL, Huisman F, Taborek P, Yu C, Kintner C. 2009. The pcp pathway instructs the planar orientation of ciliated cells in the Xenopus larval skin. Curr. Biol. 19:924929

97. Mizuno N, Taschner M, Engel BD, Lorentzen E. 2012. Structural studies of ciliary components.

J. Mol. Biol. 422:163-180

98. Najafi A, Golestanian R. 2004. Simple swimmer at low reynolds number: Three linked spheres. Phys. Rev. E 69:062901

99. Niedermayer T, Eckhardt B, Lenz P. 2008. Synchronization, phase locking, and metachronal 
wave formation in ciliary chains. Chaos 18:037128

100. Okamoto K, Nakaoka Y. 1994. Reconstitution of metachronal waves in ciliated cortical sheets of paramecium - wave stabilities. The Journal of Experimental Biology 192:61-72

101. Osterman N, Vilfan A. 2011. Finding the ciliary beating pattern with optimal efficiency. Proc. Natl. Acad. Sci. USA 108:15727-15732

102. Palagi S, Jager E, Mazzolai B, Beccai L. 2013. Propulsion of swimming microrobots inspired by metachronal waves in ciliates: from biology to material specifications. Bioinspir. Biomim. 8:046004

103. Pande J, Smith AS. 2015. Forces and shapes as determinants of micro-swimming: effect on synchronisation and the utilisation of drag. Soft Matter 11:2364-2371

104. Perkins G, Jones R. 1992. Hydrodynamic interaction of a spherical particle with a planar boundary: Ii. hard wall. Physica A 189:447-477

105. Pikovsky A, Rosenblum M, Kurths J. 2001. Synchonization. Cambridge (U.K.): Cambridge Univ. Press

106. Polin M, Tuval I, Drescher K, Gollub J, Goldstein R. 2009. Chlamydomonas swims with two "gears" in a eukaryotic version of run-and-tumble locomotion. Science 325:487 - 490

107. Pozrikidis C. 1996. Introduction to theoretical and computational fluid dynamics. Oxford University Press, USA, 1st ed.

108. Putz VB, Yeomans JM. 2009. Hydrodynamic synchronisation of model microswimmers. J. Stat. Phys. :1001-1013

109. Qian B, Jiang H, Gagnon DA, Breuer KS, Powers TR. 2009. Minimal model for synchronization induced by hydrodynamic interactions. Phys. Rev. E 80:061919

110. Reichert M, Stark H. 2004. Hydrodynamic coupling of two rotating spheres trapped in harmonic potentials. Phys. Rev. E 69:031407

111. Reichert M, Stark H. 2005. Synchronization of rotating helices by hydrodynamic interactions. Eur. Phys. J. E 17:493-500

112. Reigh SY, Winkler RG, Gompper G. 2012. Synchronization and bundling of anchored bacterial flagella. Soft Matter :4363-4372

113. Riedel-Kruse IH, Hilfinger A, Howard J, Jülicher F. 2007. How molecular motors shape the flagellar beat. HFSP Journal 1:192-208

114. Rikmenspoel R, Isles CA. 1985. Digitized precision measurements of the movements of sea urchin sperm flagella. Biophys J 47:395-410. 3978210[pmid]

115. Sanchez T, Welch D, Nicastro D, Dogic Z. 2011. Cilia-like beating of active microtubule bundles. Science 333:456-459

116. Sanderson MJ, Sleigh MA. 1981. Ciliary activity of cultured rabbit tracheal epithelium: beat pattern and metachrony. J. Cell Sci. 47:331-347

117. Sareh S, Rossiter J, Conn A, Drescher K, Goldstein RE. 2012. Swimming like algae: biomimetic soft artificial cilia. J. R. Soc. Interface

118. Satir P, Christensen ST. 2007. Overview of structure and function of mammalian cilia. Annu. Rev. Physiol. 69:377-400

119. Stubbs JL, Vladar EK, Axelrod JD, Kintner C. 2012. Multicilin promotes centriole assembly and ciliogenesis during multiciliate cell differentiation. Nat. Cell. Biol. 14:140-147

120. Tamm SL, Sonneborn TM, Dippell RV. 1975. The role of cortical orientation in the control of the direction of ciliary beat in Paramecium. J. Cell Biol. 64:98-112

121. Taylor GI. 1951. Analysis of the swimming of microscopic organisms. Proc. R. Soc. Lond. 209:447-461

122. Thornton DJ, Rousseau K, McGuckin MA. 2008. Structure and function of the polymeric mucins in airways mucus. Annu. Rev. Physiol. 70:459-86

123. Tilley AE, Walters MS, Shaykhiev R, Crystal RG. 2015. Cilia dysfunction in lung disease. Annu. Rev. Physiol. 77:379406

124. Uchida N, Golestanian R. 2010. Synchronization and collective dynamics in a carpet of mi- 
crofluidic rotors. Phys. Rev. Lett. 104:178103

125. Uchida N, Golestanian R. 2010. Synchronization in a carpet of hydrodynamically coupled rotors with random intrinsic frequency. Europhys. Lett. 89:50011

126. Uchida N, Golestanian R. 2011. Generic conditions for hydrodynamic synchronization. Phys. Rev. Lett. 106:058104

127. Uchida N, Golestanian R. 2012. Hydrodynamic synchronization between objects with cyclic rigid trajectories. Eur. Phys. J. E 35:135

128. Vilfan A, Jülicher F. 2006. Hydrodynamic flow patterns and synchronization of beating cilia. Phys. Rev. Lett. 96:058102

129. Vilfan M, Kokot G, Vilfan A, Osterman N, Kavčič B, et al. 2012. Analysis of fluid flow around a beating artificial cilium. Beilstein J. Nanotechnol. 3:163-171

130. Vilfan M, Potocnik A, Kavcic B, Osterman N, Poberaj I, et al. 2010. Self-assembled artificial cilia. Proc. Natl. Acad. Sci. USA 107:1844-1847

131. Vincensini L, Blisnick T, Bastin P. 2011. 1001 model organisms to study cilia and flagella. Biol. Cell 103:109-130

132. Wallingford JB. 2010. Planar cell polarity signaling, cilia and polarized ciliary beating. Curr. Opin. Cell Biol. 22:597-604

133. Wan KY, Leptos KC, Goldstein RE. 2014. Lag, lock, sync, slip: The many 'phases' of coupled flagella. J. R. Soc.: Interface 11:20131160

134. Werner M, Mitchell B. 2012. Understanding ciliated epithelia: The power of Xenopus. Genesis 50:176-185

135. Wollin C, Stark H. 2011. Metachronal waves in a chain of rowers with hydrodynamic interactions. Eur. Phys. J. E 34:42

136. Wong LB, Miller IF, Yeates DB. 1993. Nature of the mammalian ciliary metachronal wave. Journal of Applied Physiology 75:458-467

137. Woolley D, Vernon G. 2001. A study of helical and planar waves on sea urchin sperm flagella, with a theory of how they are generated. J. Exp. Biol. 204:1333-1345 